Supporting workers with disabilities: A scoping review of the role of human resource management in contemporary organisations

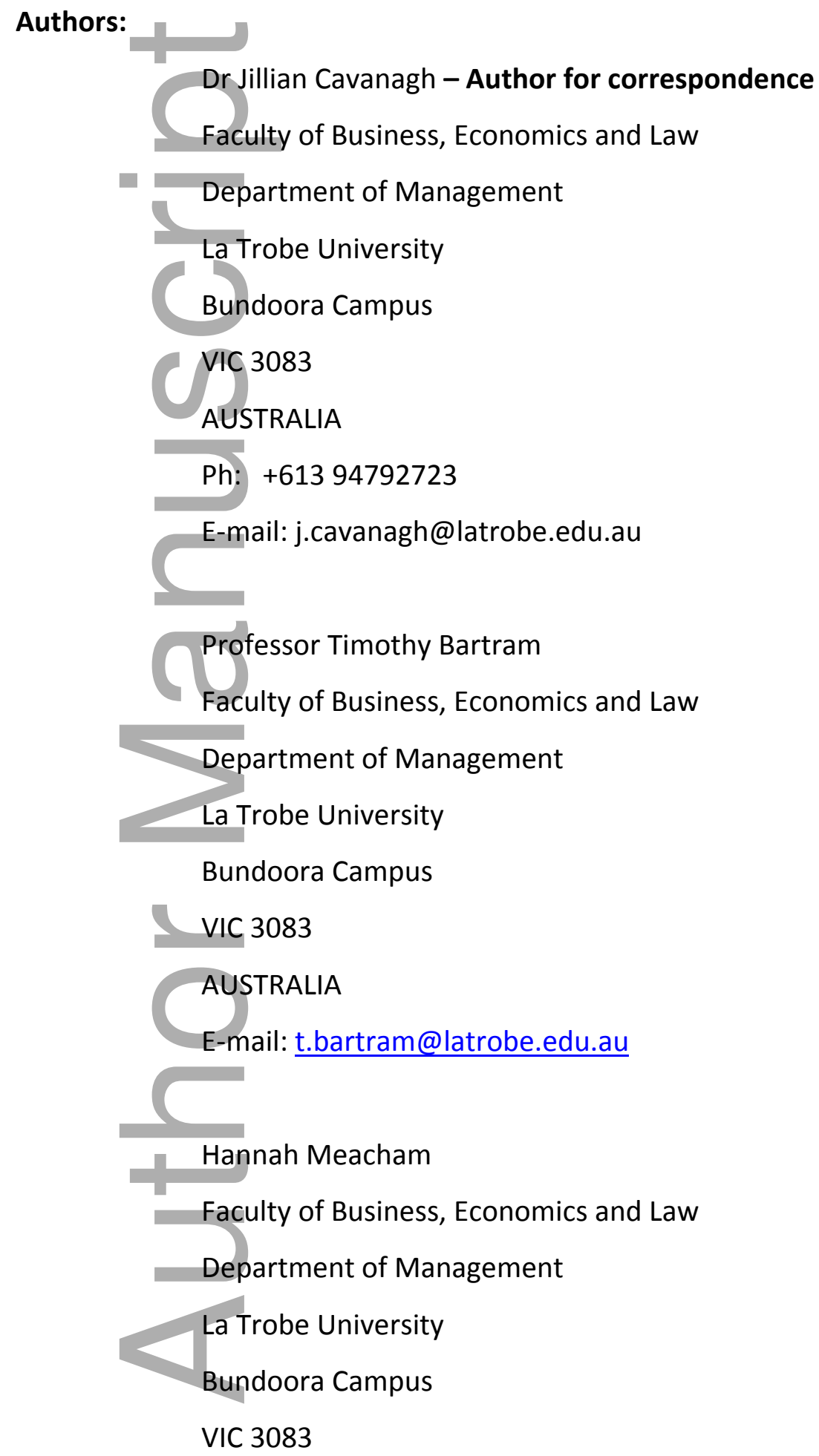

This is the author manuscript accepted for publication and has undergone full peer review but has not been through the copyediting, typesetting, pagination and proofreading process, which may lead to differences between this version and the Version of Record. Please cite this article as doi: 10.1111/aphr.12111

This article is protected by copyright. All rights reserved 
AUSTRALIA

E-mail:17642659@students.latrobe.edu.au

Professor Christine Bigby

Faculty of Health Sciences

Department of Social Work and Social Policy

La Trobe University

Bundoora Campus

VIC 3083

AUSTRALIA

E-mail: c.bigby@latrobe.edu.au

Dr Jodi Oakman

Faculty of Health Sciences

Department of Human Biosciences and Public Health

La Trobe University

Bundoora Campus

VIC 3083

AUSTRALIA

E-mail: j.oakman@latrobe.edu.au

Professor Ellie Fossey

Faculty of Health Sciences

VIC 3083

AUSTRALIA

E-mail: ellie.fossey@monash.edu.au

This article is protected by copyright. All rights reserved 
Article type : Invited Review Article
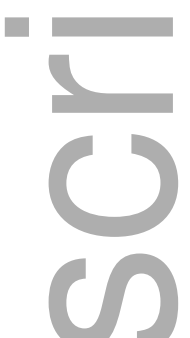

Supporting workers with disabilities: A scoping review of the role of human resource management in contemporary organisations

\begin{abstract}
This is a scoping review of literature on human resource management (HRM) and management practice that impacts on workers with physical, mental health and intellectual disabilities, employed or entering paid employment. The aim is to illuminate the use of HRM practices, managerial attitudes and employee outcomes in the disability literature. The methodological research framework commenced with seven databases and was supported with evidenced-based literature to find three main themes. Themes highlight the management and employer support for workers with disabilities, discrimination and attitudes towards employment of this cohort of workers and performance and employment outcomes. As governments around the world seek to reduce welfare costs and increase the employment of people with disabilities this paper is timely. Overall, the paper contributes to a dearth of literature on the management of people with disabilities at the workplace to unpack the key barriers, challenges and trends, and develop a comprehensive research agenda.
\end{abstract}

\title{
Introduction
}

This paper presents a scoping review of the human resource management (HRM) and disability literature to inform scholarly HRM research and management practice for workers with disability. An estimated one billon of the world's population experience some form of 
disability, of whom 785 million (15.6 per cent) are of working age (World Health Organization, 2002-2004). Yet, while people with disabilities represent a significant proportion of the potential workforce worldwide, they experience disproportionately high rates of unemployment. Average employment rates for people with disability are below 60 per cent across countries within the Organisation for Economic Co-operation and Development (OECD) (OECD, 2010). For example, in the UK, 46.3 percent of working-age people with disabilities were in employment compared to 76.4 per cent of the general working-age population (United Kingdom Government, 2012); and in Australia only 1 million of the 2.2 million working aged adults with disabilities are employed, representing 10 per cent of all Australian workers (Australian Bureau of Statistics, 2012). In most countries, workers with disability also have lower average incomes than the general workforce, with estimated differences of 15-30 per cent in earnings (OECD, 2010). Moreover, securing employment presents significant challenges for people with disabilities generally (Donelly \& Given, 2010), but it is also likely that the barriers are more challenging for some groups than others. It has been suggested that people with traumatic brain injuries are more susceptible to unemployment than any other group (Doctor et al., 2005). Given work can provide important sources of financial, social and emotional support (Lewis, Thoresen, \& Cocks, 2011), these challenges need to be addressed. Yang and Konrad (2011) argue that human resource management (HRM) practices can positively impact on employment rates of people with disabilities through enabling reasonable adjustments and the creation of more supportive workplace environments.

This paper presents a review of literature on HRM and management practices that impact current and prospective employees with disabilities. Underpinned by the literature, we develop a research agenda that calls for greater theory building and empirical research through multi-disciplinary approaches in partnership with industry and people with disabilities. Given the importance of engaging people with disabilities in meaningful work, it is hoped that this paper will encourage greater interest among researchers and management practitioners regarding the role of HRM in facilitating the employment and support of people with disabilities. The review aims to address two questions: what HR and management practices exist in contemporary organisations to support workers with physical, intellectual disabilities or mental health issues? And, how do HR and management practices impact these workers? The three categories of disability are selected and used to structure our paper as 
they represent different types of impairment which may engender different managerial approaches to HRM.

The organisation of this paper is as follows: first, we outline how disability is understood, and the method employed to undertake this review. In the subsequent sections, we present a thematic discussion of the identified literature under three themes: management and employer knowledge and support for people with disabilities; employer attitudes towards workers with disabilities; and performance and employment outcomes for employees with disabilities. We provide a syntheses of the major themes and then go on to propose an agenda for future research to serve as a guide for scholars in management and other disciplines with interests in HRM, workforce participation and disability.

\section{Disability in context}

Disability is a contested concept. Individual and social models of disability emphasize different factors, both in seeking to understand the nature of disability, and to conceptualise what is necessary to support the occupational, social and community participation of people with disabilities (Bigby \& Frawley, 2010).

The World Health Organization's (WHO) International Classification of Functioning (ICF) (2001) is a meta-model derived from individual and social models of disability, which provides a framework that is understood across professions and national boundaries. It suggests three coexisting and complimentary foci for understanding disability, and thus for adjusting work situations to take account of the needs of people with disability: capacity to function (the body and impairments); activity limitations (in executing specific actions or skills); and restrictions on participation (opportunity for involvement). In regard to employment, a focus on capacity alerts management to factors such as the health, intellectual, sensory or physical capacity of people, and the need to make workplace adjustments that take account of an individual's impaired capacity. A focus on activity is concerned with the extent to which a person's ability to undertake work-related tasks is limited by whether or not s/he can execute the actions involved (e.g., holding or lifting objects), for which reasonable adjustments may also be made to enable task performance in a different way. The participation perspective locates restrictions in social processes and structures rather than the individual. In the case of a person's job, these participation restrictions might include management processes that do not readily take into account the particular needs of a person with an intellectual, sensory or physical impairment or the negative attitudes of other 
workers, managers and staff in employment placement agencies toward workers with disabilities (Fyffe, 2007).

Commonly, in the literature, the term disability is used to refer to what, in the ICF model (WHO, 2001) is termed an impairment. While much literature refers to people with disabilities collectively, many studies focus on people with particular types of impairment. In this paper our focus is on three particular groups of people who experience disability in employment; those with physical impairment, those with mental health issues and those with intellectual impairment. For consistency and clarity we use the terms commonly used in the literature; physical disability, people with mental health problems and intellectual disability.

Increasing the economic participation of people with disabilities has been central to social policies of many western countries (International Labour Office 2012). In 2006 the United Nations Convention on the Rights of Persons with Disabilities (2006) encapsulated these aims in Article 7 mandating that every individual with a disability has a right to work on the same basis as their non-disabled peers. Australia is a signatory to the convention and the commitment to the economic participation of people with disabilities is set out in the National Disability Strategy. A range of strategies have been used throughout the world to implement such policy aims including: anti-discrimination legislation that requires employers to make reasonable adjustments; quotas for the number of people with disabilities to be employed by large organisations; subsidies to employers to partially cover the wage costs of employing a person with a disability; disability action plans within organisations to ensure processes and structures are accessible and responsive to the needs of people with disabilities; specialist disability employment services; and varied approaches to the provision of employment programs and support (e.g., enclaves, supported employment, place and train, co-worker training). Since many of these interventions rely on the knowledge and support of human resource (HR) and other managers throughout organisational hierarchies, it is important to understand what HRM and management practices are most useful in supporting workers with disabilities

\section{Literature review approach, quality and categorisation of articles}

This literature review was conducted in three stages. First, we conducted searches of the 7 databases listed in Table 1. Our search was delimited to refereed journal articles from the year 2000 to present to consider literature that impacts on contemporary workers. We used 
search terms including 'employees with disabilities', 'disabled employee', 'disabled job applicant', 'handicap management', 'handicap best practice', 'handicap effective management', 'handicap performance management' and 'handicap productive and success and management'. This search resulted in 1078 articles.

Table 1 Effective management practices and workers with disabilities

\begin{tabular}{|l|l|}
\hline Database & Results \\
\hline PsycINFO & 212 \\
\hline CINAHL & 407 \\
\hline Informit Complete & 15 \\
\hline Business Source Complete (A) & 20 \\
\hline Business Source Complete (B) & 225 \\
\hline ABI/Inform (A) & 82 \\
\hline ABI/Inform (B) & 117 \\
\hline
\end{tabular}

As a team, we systematically reviewed each article, eliminating any not considered relevant to management. For instance if an article was about rehabilitation and did not mention management and how a worker was rehabilitated we discounted the article. This reduced the total number to 264. In the second stage, an ancillary search was conducted using Advanced Google Scholar and Proquest and a hand search was undertaken of each article's reference list. Eighteen additional articles were identified, bringing the total to 282 .

In the third stage, an annotated bibliography, was compiled with a summary of each of the articles which were then categorised according to the primary group of people on which it focused: people with physical disability, people with intellectual disability, or people with mental health problems. We identified and mapped themes across the 282 articles and s to complete the review and develop three tables. Importantly, we also conducted a quality assessment of selected articles included in our review. Following the work of Tranfield, Denyer and Smart (2003) we examined a sample of research quality in terms of journal outlet, data quality, sampling strategy and theoretical adequacy. It was clear that the vast majority of research articles examining HRM and disability have not been published in high quality research outlets (as measured by impact factor and various international journal ranking schemes). For example, we examined 16 management journals; this represented all of 
the disability literature published within this specific discipline. Consistent with the Australian Business Dean's Council (ABDC) journal listing, two of the journals, 'Human Resource Management' and the 'International Journal of Hospitality Management', have ranked ' $\mathrm{A}$ *', six journals rank ' $\mathrm{A}$ ', two 'B', two ' $\mathrm{C}$ ' and four are unranked. In accordance with the United Kingdom based Cranfield School of Management Journal Rankings system 'Human Resource Management' and 'Work, Employment and Society' rank '4' (being the highest), three journals rank ' 3 ', two rank ' 2 ', two ' 1 ' and six are unranked. The impact factor, a calculation of the frequency of author citations across ISI ranked journals, has also been used to assess the quality of the 16 management journals. The 'International Journal of Hospitality Management' 1.939 records the highest impact factor across these journals with the 'British Journal of Management' recording 1.584, followed by 'Work and Occupations' 1.222. The vast majority of articles in management journals utilise small convenience samples which attempt to examine current disability employment practice. Twenty-five of the thirty-three management studies (including literature reviews) were qualitative and only eight were medium to large-scale quantitative studies. The majority of articles do not attempt to advance our theoretical understanding of HRM and disability but explicitly establish current management practice in a particular context. Fifty-two of the articles were published in nonmanagement journals, particularly from the health, psychology, law, tax and rehabilitation fields. Although these articles make important contributions they have similar limitations to the aforementioned management studies in terms of journal quality, research methodology and theoretical advancement of understanding management and disability.

Three common themes emerged: 1) Management and employer knowledge and support for people with disabilities; 2) Discrimination and attitudes towards employment of people with disabilities; and 3) Performance and employment outcomes of workers with disabilities. Each theme is presented, followed by issues specific to each of the three disability groups on which this review has focused. We provide in text tables of the literature reviewed as per the three aforementioned key themes (See Tables 2, 3 and 4).

\section{Management and employer knowledge and support for workers with disabilities}

The literature regarding management and employer knowledge and support of people with disabilities can be broadly characterised as addressing the perceived challenges of making reasonable adjustments to accommodate people with disability; the potential for employer 
costs to increase if people with disabilities are employed; and a poor understanding of disability or appropriate HR and management support. Employers, it seems, often do not fully understand government support available for employing people with disabilities. It is clear that management responses and concerns differ depending on the type of disability. For example, HR professionals and managers frequently struggle to accommodate and manage the needs of workers with physical disabilities (Kaye, Jans, \& Jones, 2011) and appropriate management support is not always forthcoming (Barlow, Wright, \& Wright, 2003). The literature identifies a disconnection between HR policies and their implementation in practice (Kulkarni \& Valk, 2010) and fear of prohibitive costs in making workplace adjustment for workers with disabilities (Lewis, Dobbs, \& Biddle, 2013; Yang \& Konrad, 2011). Moreover, evidence suggests that managers and employers more generally have limited understanding or training regarding how to support workers with intellectual disabilities and those with mental health issues (Houtenville \& Kalargyrou, 2012). Managers are often not aware of appropriate disability management practices or effective recruitment and retention practices for people with disabilities (Cook \& Burke-Miller, 2015; Lerner et al., 2004; MacDonaldWilson, Rogers, Massaro, Lyass, \& Crean, 2002). It is clear that the mainstream HRM and disability literature does not sufficiently address the complexity surrounding disability or relationships between people with disabilities and organisations. Williams and Mavin (2012: 160) argue that the literature on disability needs to further engage 'in debating constructions of disability, impairment, ableism or disabled people as members of a hierarchy ordered by social relations'. Much of this literature is premised on the assumption that 'able-bodiedness' is the norm and invariably contributes to silencing the voice of workers with disabilities at the workplace (Mumby, 2008) .

\section{Issues specific to the employment of workers with physical disabilities}

The identified literature indicates that HR professionals and managers often perceive challenges in adjusting workplaces for workers with physical disabilities (Barlow et al., 2003; Donelly \& Given, 2010; Kulkarni \& Valk, 2010). Underpinning organisational policies and approaches to employing workers with physical disabilities do not provide adequate disability strategies. This is compounded by discriminatory recruitment and selection processes and by employers not fully aware of the needs of potential employees or available government support for hiring a person with a physical disability (Jones \& Schmidt, 2004; Kaye et al., 2011; Lewis et al., 2011). Furthermore, Hamner, Hall, Timmons, Boeltzig and Fesko (2008) 
contend that recruitment is challenging because managers often fail to work closely with employment agencies. According to Duff and Ferguson (2007) most companies invest very little in recruitment and, due to their professional ignorance, often fail to recruit employees with physical disabilities.

Groschl (2007) conducted interviews with 42 hotel HR managers across Canada and 15 employment services to investigate the effect of current HR policies on the recruitment of workers with physical disabilities. Only five of the 42 hotels had a diversity statement, none of which mentioned employees with physical disabilities, and only one hotel had a specific recruitment strategy for workers with physical disabilities. Roessler, Hurley and McMahon (2010) argue most HR professionals have little knowledge of anti-discrimination policies and are not cognisant of prevention programs aimed at anti-discrimination practices. Further, many HR practitioners are unaware of intervention strategies that promote inclusion through for example, non-discriminatory work practices or flexible work schedules. Fear also exists among HR professionals that workers with physical disabilities will become a liability due to the costs associated with making workplace adjustments (Lewis et al., 2013; Yang \& Konrad, 2011). In part, this may be because employers are not always aware of the financial help available to support workers with physical disabilities (Jasper \& Waldhart, 2013). Hence, the deficiency of organisation-wide disability awareness increases the instances of discriminatory practices against workers with physical (and other) disabilities.

Employers willing to carry out structural adjustments for workers with disabilities generally offer limited personal and professional support. This is evidenced through discriminatory practices identified in the workplace that include: the denial or delay of employee promotion (Schur, Kruse, \& Blanck, 2005); stricter standards of performance (Graffam, Shinkfield, Smith, \& Polzin, 2002); forced and unwarranted supervision and failure to appoint due to the disability (Shier, Graham, \& Jones, 2009). Hartnett, Stuart, Thurman, Loy and Batiste (2011) for example, recruited respondents from a job network of 766 various and diverse employers. The study reports that 43.9 per cent of organisations provide structural workplace adjustments, 22.9 per cent modify work schedules to allow employees with disabilities more time to adjust to work conditions, and 4.1 per cent provide formal education relative to the focus of a specific organisation. Only 3 per cent of organisations offer a mentor or personal support, 2.5 per cent claim to make some adjustments to workplace policies and 2.5 per cent provide telephone work. Unger and Kregel's (2003) study of 46 human resource 
professionals from the retail, banking, healthcare, insurance, hospitality, technology and manufacturing sectors found most managers express confidence in their capability to support employees with disabilities. However, very few have the power and resources to provide appropriate workplace adjustments for employees (Unger \& Kregel, 2003). Results demonstrate there are increasing efforts on the part of employers to offer structural modifications but minimal attention to personal development.

The lack of job-specific training offered to workers with physical disabilities is in turn reflected in their lower wages and more limited employment benefits compared to those of employees without disabilities (Schur, Kruse, Blasi, \& Blanck, 2009). Consequently, for workers with physical disabilities, Schur, Kruse, Blasi and Blanck (2009) argue that these issues promote job uncertainty, low job satisfaction, high turnover rates, and impact on employee loyalty. There is no clear evidence to suggest that the management of physical disabilities is any better than management affords workers with psychological disabilities (Roessler, Hennessey, Neath, Rumrill, \& Nissen, 2011).

Nevertheless, the rate of employment opportunities can be increased for workers with physical disabilities when there are effective management interventions in place. Barlow, Wright and Wright (2003) evaluated an employment program in the UK designed to provide advisor support to 44 job applicants of various jobs. The program was successful in increasing confidence, morale and self-esteem, and decreasing the anxiety of participants seeking employment. Lewis, Thoresen and Cocks (2011) examined the data from three research projects that conducted interviews with 150 workers with disabilities who received additional external job training between 1999 and 2009. When support is forthcoming, the majority of workers with disabilities will complete their placements at a higher level than their able-bodied counterparts. Schmidt and Smith (2007) determine that those workers with physical disabilities participating in supportive employment programs are less likely to experience or report barriers to employment.

\section{Issues specific to the employment workers with mental health issues}

Lerner et al. (2004) contend that workers with mental health issues experience lower rates of productivity, the highest rates of turnover and frequent absenteeism. Many workers with mental health issues have limited earning potential (Jones, Latreille, \& Sloane, 2006) and it appears many organisations have not implemented appropriate HRM policies or support for 
workers with mental health issues (Cunningham, James, \& Dibben, 2004; MacDonaldWilson et al., 2002). Contributing factors are likely to be a lack of knowledge, skills and abilities on the part of managers and more general cost considerations. Hand and Tryssenaar (2006) interviewed 58 managers in Canada and only 22 expressed their willingness to even consider hiring individuals with mental health disabilities. Some authors have sought to compare job tenure between individuals with mental health disabilities and the general working population.

MacDonald-Wilson, Rogers, Massaro, Lyass and Crean's (2002) conducted a multi-site qualitative study of 191 individuals with psychiatric conditions in various entry-level jobs in the retail, services, wholesale, finance and manufacturing sectors across the USA. More than half of the respondents of this study were subsequently unemployed within 12 months of appointment. Jones, Latreille and Slone (2006) examined Labour Force Survey data in the UK between 1997 and 2003, and found there was a discrepancy in wages for workers with mental health issues (and other disabilities). Workers with depression experienced the highest rate of turnover and absenteeism. Using a sample of 100 employers across the areas of education healthcare, manufacturing and technology in Beijing, Hong Kong and Chicago, Tsang, et al. (2007) found that most employers have a variety of concerns relating to employees with mental illness. Concerns surrounded the safety of other employees and customers, productivity and job performance, unpredictable behaviours, and the potential for symptom relapse.

Houtenville and Kalargyrou (2012) conducted a study of 320 hospitality employers in the US and found most employers were concerned about the lack of skills of the worker, cost implications and the inability for supervisors to successfully manage workers with mental health problems. Lerner et al. (2004) carried out a broad-ranging study in Massachusetts (USA) with 229 employees who reported depression and found that many workers claimed employers do not know how to manage this group of workers. Workers maintained they had been incorrectly diagnosed, had not been treated for depression and believe employers do not know how to manage workers with depression, which suggests they may avoid a condition that is becoming more prevalent in today's society. Moreover, workers with mental health problems often do not disclose their problems for fear losing their jobs if they do so (Charmaz, 2010). 
Analysing data from the 1998-1999 labour force survey of 30,000 employees across the UK, Almond and Healey (2003) aimed to develop management understandings of absenteeism for workers with mental health disabilities. Out of the 30,000 respondents, one per cent disclosed experiencing long term mental health problems. Out of those with a mental health disability, 16 per cent were absent from work for at least one day within the reporting week compared to four per cent of workers with no disability. Bengisu and Balta (2011) conducted a small-scale Delphi study in the field of tourism with 18 workers with disabilities. The aim of this study was to identify factors that would provide guideline areas for employers when employing a worker with a mental health disability. The main areas were identified as recruitment, and health and safety for workers, colleagues and customers/clients. Even though every participant agreed workers should be appointed based on experience and skills, 11 felt employees with mental health disabilities would always struggle to work in the hospitality sector.

More specifically, a body of research examines work places and employees with schizophrenia. Zissi, Rontos, Papageorgiou, Pierrakou, \& Chtouris (2007) surveyed 102 local employers in small-sized retail and trade businesses in the Greek Islands. They found only 28 per cent and 34 per cent of employers respectively indicated a willingness to hire workers with schizophrenia and depression. Sakai, Hashimoto and Inuo (2009) interviewed 36 schizophrenia outpatients in Japan to establish the employability factors that may increase the success of employment for people with schizophrenia. This study found that openness by a worker can increase the likelihood of positive management. Salkever et al, (2007) aimed to establish the employment outcomes for 2,300 workers with schizophrenia by analysing data from the US Schizophrenia and Assessment Program. Findings indicate that only 57 per cent of 2,300 participants were employed in fulltime employment. Earning potential for people with schizophrenia was significantly less than workers without disabilities, with 11.5 per cent of respondents reporting that they earned less that then federal minimum wage. Rosenheck et al. (2006) analysed survey data from 1400 patients in the USA who participated in a clinical trial supporting an employment intervention program for with workers with schizophrenia. It was established that supported employment facilitates the working life of a worker in an employment setting and also improves the overall quality of everyday life. For the participants of this study being employed was associated with having better mental functioning and fewer severe symptoms. 
Roush (2009) conducted a study to determine the employment outcomes of a supported Abacus Program, an employment program designed to support individuals with a severe mental health issue gain meaningful employment. The study found that of the 264 respondents 83 gained employment due to the support of 140 vocational service providers. The vocational services met critical criteria to ensure the respondents had the skills to complete job applications, attend job interviews, and receive on-going Abacus support. In a North American study Charmaz (2010) interviewed 185 workers with mental health issues, and reported that if the worker does not believe the disability will impact their job, then colleagues and managers are more likely to believe this too. Significantly, if an employee feels accepted within an organisation they are more likely to disclose a disability and seek employer or external support.

The literature relative to HR policy, management practices highlights the need to better understand the requirements and accommodations of workers with mental health disabilities (McDowell \& Fossey, 2015). HRM policies and levels of management support make it difficult for employers to employ workers with disabilities because responsibilities deviate across organisations (Cunningham et al., 2004). In one organisation there may be a HR department and in another a supervisor with no HR qualifications or experience and ad-hoc policies do not provide workers with the foundational support they need. Ball, Monaco, Schmelling, Schartz and Blank (2005) investigated the diversity policies of the top 100 profit making companies from the Fortune Magazine's top 500 in the USA. The majority of companies reported the existence of diversity policies and $40 \%$ were identified as inclusive, $47 \%$ were non-committal and $13 \%$ did not mention disability in their diversity policy (Ball et al., 2005). Ethnicity and gender were central to most policies and workers with disabilities were found to be the forgotten diversity group.

Lexen, Hofgren and Bejerholm (2013) argue management should allow sufficient time for employees to complete job tasks which will stimulate worker satisfaction and increase performance. Employers who provide support for workers with mental health issues will benefit from increased productivity (Gates, 2000; Lagerveld et al., 2010). Smith, Webber, Graffam and Wilson (2004b) surveyed 656 employers who used a disability employment service to establish the perceptions of employers of job matching and job performance of workers with disabilities. The findings indicated that using a disability employment service can be beneficial for the employer in matching candidates to specific jobs. Snyder, 
Carmichael, Blackwell, Cleveland and Thornton (2010) argue that employees with disabilities experience procedural injustices and job dissatisfaction relative to employees without disability. Moreover, Basset, Lloyd and Bassett (2001) argue whilst ever there is limited support for workers with mental health disabilities the condition will continue to be an impediment to employment. Supported employment programs are critical to ensure workers with mental illness are placed in sustainable work situations and supported throughout their employment (Roush, 2009).

\section{Issues specific to the employment of workers with intellectual disability}

There appears to be limited managerial knowledge of the hardships employees, with intellectual disabilities, experience in the workplace. Management policies and practices rarely ensure efficient processes are in place to take account of workers with intellectual disabilities (Scheid, 2005), and there is limited knowledge of the difficulties that these workers experience (Alston, Bell, \& Hampton, 2002; Price \& Gerber, 2001). According to Price and Gerber (2001) this cohort of workers receives the least management support compared to other forms of disability. Commonly, there is a deficiency of role models for these workers, which may lead to a lack of confidence when applying for jobs (Alston et al., 2002). Luecking, Cuozzo and Buchanan (2006) suggest that employers are only willing to support people with intellectual disabilities with customised work assignments when they perceive there will be a direct benefit to the organisation. From a worker's perspective their experiences are likely to be negative if managers have had no previous experience of managing an employee with an intellectual disability (Cunningham et al., 2004). Support from colleagues has a flow on effect from management and is often delayed due to policy constraints and a lack of diversity training.

Employers often fail to design and support management and HR policies that require supervisors and colleagues to devote more time and effort in assisting individuals with intellectual disabilities (Lysaght, Ouellette-Kuntz, \& Lin, 2012). Schur et al. (2005) suggest attention must be afforded to 'the ways in which corporate culture creates or reinforces obstacles to employees with disabilities and how these obstacles can be removed or overcome' (p.3). Cunningham et al. (2004) analysed four case studies across two public sector and two private sector organisations, interviewing 12 managers and 28 workers with disabilities. The study found HR responsibilities vary across organisations and ad-hoc approaches do not provide effective support for workers with intellectual disabilities. In the 
US there are indications of employer non-compliance to the Americans with Disabilities Act (ADA) (Price \& Gerber, 2001). Employers are avoiding the recruitment of workers with disabilities due to lack of knowledge and taking up support offered by agencies. This highlights a lack of understanding and limited willingness of managers to understand the needs of workers with intellectual disabilities by employers. Moreover, the lack of support by external agencies compounds the employer's excuses not to employ workers with intellectual disabilities.

Fillary and Pernice's (2006) research of 8 retail and restaurant organisations in New Zealand reveals that inclusion rates for employees with an intellectual disability is marginal. This study found that the majority of employees without an identified disability were included in more than 80 per cent of workplace culture elements, but only three workers with an intellectual disability were included. Fevre et al. (2013) surveyed 284 workers with disabilities in the UK and 52 respondents reported a psychological disability (in this study mental illness and intellectual disability were categorised as psychological disability). For those with a psychological disability, the likelihood of experiencing any ill-treatment at work increased by 177 per cent. Lysaght, Ouellette-Kuntz and Lin (2012) claim employers must promote employment opportunities by reconceptualising job design, implementing conducive recruitment practices, and establishing internal cultures of diversity.

Table 2 Management and employer knowledge and support for workers with physical, mental health and intellectual disabilities

\begin{tabular}{|c|c|c|c|c|}
\hline Author(s) & Journal & $\begin{array}{l}\text { Year of } \\
\text { Publication }\end{array}$ & $\begin{array}{l}\text { Data Collection } \\
\text { Method }\end{array}$ & Three Key Terms \\
\hline \multicolumn{5}{|l|}{ Physical Disability } \\
\hline $\begin{array}{l}\text { Barlow, J. H., Wright, C. C., \& } \\
\text { Wright, S. }\end{array}$ & $\begin{array}{l}\text { International Journal of } \\
\text { Rehabilitation Research }\end{array}$ & 2003 & $\begin{array}{l}\text { Qualitative and } \\
\text { Quantitative }\end{array}$ & Arthritis, employment, attitudes \\
\hline Donelly, M., \& Given, F. & $\begin{array}{l}\text { Work: A Journal of Prevention, } \\
\text { Assessment \& Rehabilitation }\end{array}$ & 2010 & Qualitative & $\begin{array}{l}\text { Disability, employment } \\
\text { programs }\end{array}$ \\
\hline Duff, A., \& Ferguson, J. & $\begin{array}{l}\text { Critical perspectives on } \\
\text { Accounting }\end{array}$ & 2007 & Quantitative & $\begin{array}{l}\text { Social model, emancipation, } \\
\text { accounting }\end{array}$ \\
\hline $\begin{array}{l}\text { Graffam, J., Shinkfield, A., } \\
\text { Smith, K., \& Polzin, U. }\end{array}$ & $\begin{array}{l}\text { Journal of Vocational } \\
\text { Rehabilitation }\end{array}$ & 2002 & Qualitative & $\begin{array}{l}\text { Recruitment, employment, } \\
\text { management }\end{array}$ \\
\hline Groschl, S. & $\begin{array}{l}\text { International Journal of } \\
\text { Hospitality Management }\end{array}$ & 2007 & $\begin{array}{l}\text { Qualitative and } \\
\text { Quantitative Study }\end{array}$ & Policy, integration, tourism \\
\hline $\begin{array}{l}\text { Hamner, D., Hall, A. C., } \\
\text { Timmons, J. C., Boeltzig, H., \& } \\
\text { Fesko, S. }\end{array}$ & $\begin{array}{l}\text { Journal of Organizational Change } \\
\text { Management }\end{array}$ & 2008 & Qualitative & Support, employment, change \\
\hline Hartnett, H. P., Stuart, H., & Journal of Vocational & 2011 & Qualitative and & Accommodation benefits, social \\
\hline
\end{tabular}




\begin{tabular}{|c|c|c|c|c|}
\hline $\begin{array}{l}\text { Thurman, H., Loy, B., \& Batiste, } \\
\text { C. }\end{array}$ & Rehabilitation & & Quantitative & service \\
\hline Jasper, C. R., \& Waldhart, P. & $\begin{array}{l}\text { International Journal of } \\
\text { Contemporary Hospitality } \\
\text { Management }\end{array}$ & 2013 & Qualitative & $\begin{array}{l}\text { Recruitment, hospitality, } \\
\text { attitudes }\end{array}$ \\
\hline Jones, P., \& Schmidt, R. A. & $\begin{array}{l}\text { International Journal of Retail \& } \\
\text { Distribution Management }\end{array}$ & 2004 & Literature Review & Retail industry, disability \\
\hline $\begin{array}{l}\text { Kaye, H. S., Jans, L. H., \& } \\
\text { Jones, E. C. }\end{array}$ & $\begin{array}{l}\text { Journal of Occupational } \\
\text { Rehabilitation }\end{array}$ & 2011 & Quantitative & $\begin{array}{l}\text { Employment, employer } \\
\text { attitudes, discrimination }\end{array}$ \\
\hline Kulkarni, M., \& Valk, R. & IIMB Management Review & 2010 & Qualitative & $\begin{array}{l}\text { People with disabilities, human } \\
\text { resource practices }\end{array}$ \\
\hline $\begin{array}{l}\text { Lewis, G., Thoresen, S. H., \& } \\
\text { Cocks, E. }\end{array}$ & $\begin{array}{l}\text { Journal of Vocational } \\
\text { Rehabilitation }\end{array}$ & 2011 & Quantitative & Trainees, support, placement \\
\hline $\begin{array}{l}\text { Lewis, R., Dobbs, L., \& Biddle, } \\
\text { P. }\end{array}$ & Disability \& Society & 2013 & Qualitative & Employment support, attitudes \\
\hline $\begin{array}{l}\text { Roessler, R., Hennessey, M., } \\
\text { Neath, J., Rumrill, P., \& Nissen, } \\
\text { S. }\end{array}$ & Journal of Rehabilitation & 2011 & Qualitative & $\begin{array}{l}\text { Multiple sclerosis, employment, } \\
\text { discrimination }\end{array}$ \\
\hline $\begin{array}{l}\text { Roessler, R. T., Hurley, J. E., \& } \\
\text { McMahon, B. T. }\end{array}$ & $\begin{array}{l}\text { Advances in Developing Human } \\
\text { Resources }\end{array}$ & 2010 & Previous data (records) & $\begin{array}{l}\text { Termination, discrimination; } \\
\text { disability }\end{array}$ \\
\hline Schmidt, M.A., \& Smith, D.L. & $\begin{array}{l}\text { Work: A Journal of Prevention, } \\
\text { Assessment \& Rehabilitation }\end{array}$ & 2007 & Qualitative & $\begin{array}{l}\text { Employment, recruitment, work } \\
\text { readiness }\end{array}$ \\
\hline $\begin{array}{l}\text { Shier, M., Graham, J. R., \& } \\
\text { Jones, M. E. }\end{array}$ & Disability \& Society & 2009 & Qualitative & $\begin{array}{l}\text { Employment, disability, } \\
\text { discrimination }\end{array}$ \\
\hline $\begin{array}{l}\text { Schur, L., Kruse, D., \& Blanck, } \\
\text { P }\end{array}$ & Behavioural Sciences \& the Law & 2005 & Literature Review & $\begin{array}{l}\text { Employment, culture, } \\
\text { management }\end{array}$ \\
\hline $\begin{array}{l}\text { Schur, L., Kruse, D., Blasi, J., \& } \\
\text { Blanck, P. }\end{array}$ & Industrial Relations & 2009 & Quantitative & $\begin{array}{l}\text { Corporate culture, job security, } \\
\text { promotions }\end{array}$ \\
\hline Unger, D. \& Kregel, J. & $\begin{array}{l}\text { Work: A Journal of Prevention, } \\
\text { Assessment \& Rehabilitation }\end{array}$ & 2003 & $\begin{array}{l}\text { Qualitative and } \\
\text { Quantitative }\end{array}$ & $\begin{array}{l}\text { Accommodations, workplace } \\
\text { support }\end{array}$ \\
\hline Yang, Y., \& Konrad, A. I. M. & $\begin{array}{l}\text { Group \& Organization } \\
\text { Management }\end{array}$ & 2011 & Qualitative & $\begin{array}{l}\text { Diversity, management policy, } \\
\text { disability }\end{array}$ \\
\hline \multicolumn{5}{|l|}{ Mental Health Issues } \\
\hline Almond, S., \& Healey, & Work Employment and Society & 2003 & Quantitative & $\begin{array}{l}\text { Absence management, labour } \\
\text { force survey, sickness }\end{array}$ \\
\hline $\begin{array}{l}\text { Ball, P., Monaco, G., } \\
\text { Schmeling, J., Schartz, H., } \\
\text { Blanck, P. }\end{array}$ & Behavioural Sciences \& the Law & 2005 & Qualitative & $\begin{array}{l}\text { Workplace diversity, } \\
\text { organizational policies, } \\
\text { discrimination }\end{array}$ \\
\hline $\begin{array}{l}\text { Bassett, J., Lloyd, C., \& Bassett, } \\
\text { H. }\end{array}$ & $\begin{array}{l}\text { British Journal of Occupational } \\
\text { Therapy }\end{array}$ & 2001 & Qualitative & $\begin{array}{l}\text { Young people, barriers, } \\
\text { psychosis }\end{array}$ \\
\hline $\begin{array}{l}\text { Bengisu, M., } \\
\text { Balta, S. }\end{array}$ & Canadian Tax Journal & 2011 & Qualitative & $\begin{array}{l}\text { Employment, corporate social } \\
\text { responsibility, diversification }\end{array}$ \\
\hline Charmaz, K. & $\begin{array}{l}\text { Journal of International Education } \\
\text { in Business }\end{array}$ & 2010 & Qualitative & $\begin{array}{l}\text { Disclosure, employee attitudes, } \\
\text { employer attitudes }\end{array}$ \\
\hline Cook, J., Burke-Miller, J. & $\begin{array}{l}\text { Journal of Rehabilitation Research } \\
\text { \& Development }\end{array}$ & 2015 & Qualitative & Job separations, mental health \\
\hline $\begin{array}{l}\text { Cunningham, I., } \\
\text { James, P., Dibben, P. }\end{array}$ & British Journal of Management & 2004 & Qualitative & $\begin{array}{l}\text { Organizational policy, } \\
\text { employment security, working }\end{array}$ \\
\hline
\end{tabular}




\begin{tabular}{|c|c|c|c|c|}
\hline & & & & conditions \\
\hline Hand, C., \& Tryssenaar, J. & Psychiatric Rehabilitation Journal & 2006 & Quantitative & $\begin{array}{l}\text { Recruitment, mental illness, } \\
\text { employers views }\end{array}$ \\
\hline $\begin{array}{l}\text { Houtenville, A., } \\
\text { Kalargyrou, V. }\end{array}$ & Cornell Hospitality Quarterly & 2012 & Qualitative & $\begin{array}{l}\text { Job skills, recruitment, } \\
\text { performance }\end{array}$ \\
\hline $\begin{array}{l}\text { Jones, M. K., } \\
\text { Latreille, P. L., } \\
\text { Sloane, P. J. }\end{array}$ & Oxford Economic Papers & 2006 & Quantitative & $\begin{array}{l}\text { Disability, gender differences, } \\
\text { legislation }\end{array}$ \\
\hline $\begin{array}{l}\text { Lagerveld, S. E., Bültmann, U., } \\
\text { Franche, R. L., van Dijk, F. J., } \\
\text { Vlasveld, M. C., van der Feltz- } \\
\text { Cornelis, C. M., Bruinvels, D. J., } \\
\text { Huijs, J. J., Blonk, R. W., van } \\
\text { der Klink, J. J., Nieuwenhuijsen, } \\
\text { K. }\end{array}$ & $\begin{array}{l}\text { Journal of Occupational } \\
\text { Rehabilitation }\end{array}$ & 2010 & Literature review & $\begin{array}{l}\text { Occupational health and safety, } \\
\text { depression, sick leave }\end{array}$ \\
\hline $\begin{array}{l}\text { Lerner, D., Adler, D. A., Chang, } \\
\text { H., Lapitsky, L., Hood, M. Y., } \\
\text { Perissinotto, C., Reed, J., } \\
\text { McLaughlin, T. J., Berndt, E. R., } \\
\text { Rogers, W. H }\end{array}$ & Psychiatric Services & 2004 & Quantitative & $\begin{array}{l}\text { Unemployment, productivity, } \\
\text { depression }\end{array}$ \\
\hline $\begin{array}{l}\text { Lexen, A., Hofgren, C., \& } \\
\text { Bejerholm, U. }\end{array}$ & $\begin{array}{l}\text { Scandinavian Journal of } \\
\text { Occupational Therapy }\end{array}$ & 2013 & $\begin{array}{l}\text { Quantitative and } \\
\text { qualitative }\end{array}$ & $\begin{array}{l}\text { Vocational rehabilitation, } \\
\text { occupational therapy, individual } \\
\text { placement }\end{array}$ \\
\hline $\begin{array}{l}\text { MacDonald-Wilson, K. L., } \\
\text { Rogers, E. S., Massaro, J. M., } \\
\text { Lyass, A., \& Crean, T. }\end{array}$ & Community Mental health Journal & 2002 & Quantitative & $\begin{array}{l}\text { Accommodations, supported } \\
\text { employment }\end{array}$ \\
\hline McDowell, C., Fossey, E. & $\begin{array}{l}\text { Journal of occupational } \\
\text { rehabilitation }\end{array}$ & 2015 & Scoping review & Accommodations, mental illness \\
\hline $\begin{array}{l}\text { Rosenheck, R., Leslie, D., } \\
\text { Keefe, R., McEvoy, J., Swartz, } \\
\text { M., Perkins, D., Stroup, S., } \\
\text { Hsiao, J. K., Lieberman, J. }\end{array}$ & American Journal of Psychiatry & 2006 & $\begin{array}{l}\text { Quantitative and } \\
\text { Qualitative }\end{array}$ & $\begin{array}{l}\text { Rehabilitation, motivation, } \\
\text { prejudice }\end{array}$ \\
\hline Roush, S. & $\begin{array}{l}\text { Work: A Journal of Prevention, } \\
\text { Assessment \& Rehabilitation }\end{array}$ & 2009 & Quantitative & $\begin{array}{l}\text { Vocational rehabilitation, menu } \\
\text { approach, supported } \\
\text { employment }\end{array}$ \\
\hline $\begin{array}{l}\text { Sakai, K., Hashimoto, T., } \\
\text { Inuo, S. }\end{array}$ & $\begin{array}{l}\text { Work: A Journal of Prevention, } \\
\text { Assessment \& Rehabilitation }\end{array}$ & 2009 & Quantitative & $\begin{array}{l}\text { Occupational health and safety, } \\
\text { vocational rehabilitation }\end{array}$ \\
\hline $\begin{array}{l}\text { Salkever, D. S., Karakus, M. C., } \\
\text { Slade, E. P., Harding, C. M., } \\
\text { Hough, R. L., Rosenheck, R. A., } \\
\text { Swartz, M. S., Barrio, C., } \\
\text { Yamada, A. M. }\end{array}$ & Psychiatric Services & 2007 & $\begin{array}{l}\text { Qualitative and } \\
\text { Quantitative }\end{array}$ & $\begin{array}{l}\text { Community-institutional } \\
\text { relations, fringe benefits, } \\
\text { schizophrenia }\end{array}$ \\
\hline $\begin{array}{l}\text { Snyder, L. A., Carmichael, J. S., } \\
\text { Blackwell, L. V., Cleveland, J. } \\
\text { N., \& Thornton III, G. C. }\end{array}$ & $\begin{array}{l}\text { Employee Responsibilities and } \\
\text { Rights Journal }\end{array}$ & 2010 & Quantitative & $\begin{array}{l}\text { Organizational justice, } \\
\text { discrimination, attitudes }\end{array}$ \\
\hline $\begin{array}{l}\text { Smith, K., Webber, L., Graffam, } \\
\text { J., Wilson, C. }\end{array}$ & $\begin{array}{l}\text { Journal of Vocational } \\
\text { Rehabilitation }\end{array}$ & 2004 & Quantitative & $\begin{array}{l}\text { Employer satisfaction, strategic } \\
\text { planning, job performance }\end{array}$ \\
\hline Tsang, H. W. H., Angell, B., & Social Psychiatry and Psychiatric & 2007 & Qualitative & Psychotic disorder, cross- \\
\hline
\end{tabular}




\begin{tabular}{|c|c|c|c|c|}
\hline $\begin{array}{l}\text { Corrigan, P. W., Lee, Y., Shi, } \\
\text { K., Lam, C. S., Jin, S., \& Fung, } \\
\text { K. M. T. }\end{array}$ & Epidemiology & & & cultural differences, lay theory \\
\hline $\begin{array}{l}\text { Zissi, A., Rontos, C., } \\
\text { Papageorgiou, D., Pierrakou, C., } \\
\text { \& Chtouris, S. }\end{array}$ & $\begin{array}{l}\text { Scandinavian Journal of Disability } \\
\text { Research }\end{array}$ & 2007 & Quantitative & $\begin{array}{l}\text { Greek employer; disability; } \\
\text { employment }\end{array}$ \\
\hline \multicolumn{5}{|l|}{ Intellectual Disability } \\
\hline $\begin{array}{l}\text { Alston, R., } \\
\text { Bell, T., Hampton, J. }\end{array}$ & Journal of Career Development & 2002 & Qualitative & $\begin{array}{l}\text { Job creation, career } \\
\text { development, learning } \\
\text { disabilities }\end{array}$ \\
\hline $\begin{array}{l}\text { Cunningham, I., } \\
\text { James, P., Dibben, P. }\end{array}$ & British Journal of Management & 2004 & Qualitative & $\begin{array}{l}\text { Organizational policy, } \\
\text { employment security, } \\
\text { working conditions }\end{array}$ \\
\hline $\begin{array}{l}\text { Fevre, R., Robinson, A., } \\
\text { D., \& Jones, T. }\end{array}$ & Work Employment and Society & 2013 & Qualitative & $\begin{array}{l}\text { Discrimination, treatment, } \\
\text { management }\end{array}$ \\
\hline Fillary, R., \& Pernice, & $\begin{array}{l}\text { International Journal of } \\
\text { Rehabilitation Research }\end{array}$ & 2006 & Quantitative & $\begin{array}{l}\text { Social inclusion, workplace } \\
\text { culture; supported employment }\end{array}$ \\
\hline Groschl, S. & $\begin{array}{l}\text { Cornell Hotel \& Restaurant } \\
\text { Administration Quarterly }\end{array}$ & 2005 & Qualitative & $\begin{array}{l}\text { Hotels, recruitment, human } \\
\text { resource management }\end{array}$ \\
\hline $\begin{array}{l}\text { Luecking, R. G., Cuozzo, L., \& } \\
\text { Buchanan, L. }\end{array}$ & $\begin{array}{l}\text { Journal of Applied Rehabilitation } \\
\text { Counseling }\end{array}$ & 2006 & Qualitative & $\begin{array}{l}\text { Demand-side workforce needs, } \\
\text { job customization }\end{array}$ \\
\hline $\begin{array}{l}\text { Lysaght, R., Ouellette-K } \\
\text { H., \& Lin, C-J. }\end{array}$ & $\begin{array}{l}\text { Work: A Journal of Prevention, } \\
\text { Assessment \& Rehabilitation }\end{array}$ & 2012 & Literature review & $\begin{array}{l}\text { Organizational behaviour, } \\
\text { inclusion, supported } \\
\text { employment }\end{array}$ \\
\hline $\begin{array}{l}\text { Price, L. A. } \\
\text { Gerber, P. J. }\end{array}$ & Journal of Learning Disabilities & 2001 & Qualitative & $\begin{array}{l}\text { Employer knowledge, disability } \\
\text { management, employer attitudes }\end{array}$ \\
\hline Scheid, T. L. & $\begin{array}{l}\text { International Journal of Law and } \\
\text { Psychiatry }\end{array}$ & 2005 & $\begin{array}{l}\text { Qualitative and } \\
\text { Quantitative }\end{array}$ & $\begin{array}{l}\text { Employer attitudes, social } \\
\text { stigma, employment barriers }\end{array}$ \\
\hline $\begin{array}{l}\text { Schur, L., Kruse, D., \& Blanck, } \\
\text { P }\end{array}$ & Behavioural Sciences \& the Law & 2005 & Literature Review & $\begin{array}{l}\text { Employment, culture, } \\
\text { management }\end{array}$ \\
\hline
\end{tabular}

\section{Discrimination and attitudes towards employment of workers with disabilities}

Research indicates that managers may hold discriminatory attitudes towards the employment of people with disabilities. Managers may perceive people with disabilities to be poor performers or to lack the necessary competencies to be effective at work (Jones \& Schmidt, 2004). In the absence of appropriate leadership or interaction with people with disabilities, other workers within organisations may hold discriminatory attitudes. However, according to Hashim and Saodah (2014), with effective organisational leadership, education and interaction with people with disabilities, stigmatising and discriminatory attitudes in the workplace can be changed. Organisation study theorists have also contributed to understanding the genesis of these discriminatory attitudes largely through critical examination of the intersection of difference, inequality and power (Tatli and Ozbilgin, 2012; Williams and Mavin, 2012). For example, Tatli and Ozbilgin (2012: 191) argue that the 
Bourdieu's theory of capitals (i.e., resources) 'has great explanatory power in understanding the construction of privilege' and our understanding of 'creating and sustaining privilege and disadvantage'. In line with Tatli and Ozbilgin (2012: 192-193) we suggest that scholarship in disability studies would benefit from 'exploring the ways in which ownership and distribution of different forms of capital between different groups and individual actors creates intersecting inequalities and privilege in organisation settings'.

\section{Discrimination and attitudes towards workers with physical disabilities}

The literature indicates that the attitudes of managers and staff in organisations towards employees with physical disabilities are difficult to determine let alone regulate (Chan et al., 2010). Employers often portray workers with physical disabilities as having low productivity rates (Jones \& Sehmidt, 2004) and stereotype them as incompetent (Schur et al., 2005) which negatively affects the attitudes of their colleagues (Cubero, 2007). Shier, Graham, and Jones (2009) argue that unfavourable attitudes can lead to instances of employer and co-worker discrimination. Balser (2002) suggests that HRM disability strategies are mostly rhetorical, with managers generally unable to deal with discrimination towards workers with physical disabilities. Moreover, Lengnick-Hall, Gaunt and Kulkarni (2008) contend that managers often fail to take a proactive approach to such issues. Jones and Schmidt (2004) for example, posit that many companies promote stereotyping and managers lack commitment to the employment of people with physical disabilities. They argue instances of hierarchical arrogance impacts on an employee's ability to perform, which exacerbates negative perceptions. Lengnick-Hall, Gaunt and Kulkarni (2008) contend employers are concerned about how other able-bodied staff will react to staff with physical disability which influences their attitudes. However, it appears that if the worker themselves believes that their physical disability will not negatively impact on their performance then colleagues and managers are likely to be in agreement (Charmaz, 2010).

Roessler et al. (2011) conducted a survey of 200 workers with multiple sclerosis (MS) in the USA. The study and found 29.5 per cent of the participants experienced workplace discrimination related to failure to make workplace structural adjustments, unfair work conditions, and punitive performance measures. Workers were expected to complete menial tasks, which impacted on their competitive right to apply for promotion. Some workers reported they were blatantly rejected for promotion and others claimed promotion was unfairly deferred. Nafukho, Roessler and Kacirek (2010) assert that HR professionals need to 
minimise workplace misconceptions about people with disabilities and disseminate positive practices that will provide all workers with a positive employment experience and a pathway to promotion.

Many organisations face challenges in the accommodation of people with physical disabilities. Perry, Hendricks and Broadbent (2000) argue that job applicants in wheelchairs experience more discrimination than those not in wheelchairs. Lee (2003) postulates the Americans with Disabilities Act (ADA) has failed in its promise to provide more employment opportunities and legislation needs to be amended to fully support workers with physical (and other) disabilities in their plight to gain employment. Rumrill and Fitzgerald (2010) undertook a review of allegations filed under the ADA between 1992 and 2008. The study found that the prevalence of discrimination and involuntary termination was in the manufacturing sector and related to unreasonable workplace accommodations, constructive dismissal, harassment and intimidation and unfair employment conditions.

Jones and Schmidt (2004) examined the Disability Discrimination Act in the UK and suggest that legislation often inhibits small organisations' compliance with requirements to make reasonable adjustments for workers with a physical disability mainly because they find the costs to be excessive and cannot justify business returns. This is not an excuse for employers to have negative attitudes towards employees with a physical disability but explains one of the constraints of small companies. Jasper and Waldhart's (2013) main concern in the hospitality industry relates to poor management attitudes and low levels of motivation of managers to employ people with a physical disability. These authors' survey of 320 leisure and hospitality companies and found that only 22.8 per cent of organisations actively engage in recruiting workers with disabilities. Kontosh, Fletcher, Frain and Winland-Brown (2007) distributed 504 surveys to registered nurses, on a Department of Health database, in a southeastern state of the United States. Usable results from 121 respondents revealed that only those nurses who had previously worked with a nurse with a physical disability had a sense of collegiality towards this group. In contrast, Hashim and Saodah (2014) sourced a list of companies through the Society of the Orthopedically Handicapped Malaysia and distributed questionnaires to employers, workers with disabilities and other employees. The final sample of 785 comprised 195 employers, 384 workers with physical disabilities and 206 colleagues. Overall, the findings showed that employers believed they treated workers with physical disabilities well, colleagues reported an inclusive work environment and helpful management 
attitudes. The respondents with physical disabilities confirmed these positive attitudes and reported they felt supported by both colleagues and employers. When employers and colleagues had previous involvement with people with physical disabilities, they were more likely to hire and integrate workers with disabilities (Hernandez et al., 2012). When employers have positive attitudes towards employees with physical disabilities they are more likely to support them to achieve their career goals (Hashim \& Saodah, 2014), which may lead to career advancement (Schroedel \& Geyer, 2001).

\section{Discrimination and attitudes towards workers with mental health issues}

Employees with mental health issues report more negative workplace experiences than employees with physical disabilities (Snyder et al., 2010). Negative attitudes often impact on the integration of workers into the organisation (Cubero, 2007). According to Scheid (2005), 'one of the most critical barriers to the employment of individuals with mental disabilities (sic) is the degree of social stigma such disabilities incur' (p.670). This accounts for one reason why workers cannot find or retain employment (Krupa, Kirsh, Cockburn, \& Gewurtz, 2009; Schulze \& Angermeyer, 2003; Zissi et al., 2007). In particular, stigma associated with schizophrenia is one of the most common barriers to employment (Schulze \& Angermeyer, 2003; Zissi et al., 2007).

Snyder, Carmichael, Blackwell, Cleveland and Thornton III (2010) found in their survey of 1,880 American university employees that workers with mental health problems were more prone to overt and subtle discrimination, as well as procedural injustices. The majority of these workers were direct in conveying to their employers the impact of workplace inequalities. Hand and Tryssenaar's (2006) study suggests that employers should be educated about mental illness in an effort to promote organisational awareness. Moreover, cultural change lead by HR departments, in partnership with all organisational participants, is necessary if attitudes towards workers with mental health issues are to improve (Schur et al., 2005).

\section{Discrimination and attitudes towards workers with intellectual disability}

The main themes in the literature about attitudes to people with intellectual disabilities relate to negative management and co-worker perceptions. Employer attitudes towards workers with intellectual disabilities are often indolent concerning this cohort of workers and create a barrier to employment (Cimera, 2010; Cimera \& Cowan, 2009; Negri, 2009; Rao, Horton, 
Tsang, Kan, \& Corrigan, 2010). Given negative employer views, Luecking, Cuozzo and Buchanan (2006) suggest that employment support programmes also fail to promote the benefits of employing workers with an intellectual disability. Employees with an intellectual disability have been found to be less likely to be socially accepted by colleagues than workers with physical disabilities (Loo, 2004). This is related to the social stigma often attached to intellectual disability (McLaughlin, Bell, \& Stringer, 2004).

Hoque et al. (2014) analysed survey data from 116 trade union disability champions under the 'two ticks' system in the UK. The system sets goals for organisations to adhere to in regard to supporting workers with disabilities. The main aim of the study was to assess the willingness of employers using the 'two ticks' symbol to support trade union disability champions. Forty-three employers reported that employees were given reasonable time off to complete their disability champion role but suggested there was inadequate support from government for the initiative. Government policy, training and employer attitudes need to change in order to improve employment prospects of workers with intellectual disabilities (Shaw, Jacobs, Lysaght, Ouellette-Kuntz, \& Lin, 2012). Similarly, Lantz and Marston (2012) contend that in Australia the Welfare to Work Reforms in 2006 do not fully support workers with intellectual disabilities and they offer insufficient support to assist with gaining meaningful employment.

Employer and colleague attitudes can greatly influence the well-being of workers with disabilities (Cubero, 2007). McLaughlin, Bell and Stringer (2004) examined the social inclusion of 643 students enrolled in undergraduate organisational behaviour courses in a south-western USA university. Participants were given questionnaires depicting a scenario of an employee with either AIDS, cerebral palsy or the consequences of a stroke. It was found that participants were more likely to socially include a worker with a stroke due to empathy and understanding of the disability, compared to someone with either AIDS or cerebral palsy. The stigma associated with particular conditions and stereotypical views often influence judgements of managers and colleagues about the ability of workers with disabilities, and their willingness to interact socially with them (McLaughlin et al., 2004).

Table 3 Discrimination and attitudes towards employment of workers with disabilities

\begin{tabular}{|l|l|l|l|l|}
\hline Author(s) & Journal & $\begin{array}{l}\text { Year of } \\
\text { Publication }\end{array}$ & Data Collection Method & Three Key Terms \\
\hline Physical Disability
\end{tabular}




\begin{tabular}{|c|c|c|c|c|}
\hline Balser, D. B. & Work and Occupations & 2002 & Empirical Study & Discrimination, organisation behaviour \\
\hline $\begin{array}{l}\text { Chan, F., Strauser, D., } \\
\text { Maher, P., Lee, E., Jones, } \\
\text { R., Johnson, E. T. }\end{array}$ & $\begin{array}{l}\text { Journal of Occupational } \\
\text { Rehabilitation }\end{array}$ & 2010 & Quantitative & $\begin{array}{l}\text { Attitudes, rehabilitation, vocational, } \\
\text { selection }\end{array}$ \\
\hline Charmaz, K. & $\begin{array}{l}\text { Journal of International } \\
\text { Education in Business }\end{array}$ & 2010 & Qualitative & $\begin{array}{l}\text { Disclosure, employer attitudes, work } \\
\text { environment }\end{array}$ \\
\hline Cubero, G. & $\begin{array}{l}\text { Work: A Journal of } \\
\text { Prevention, Assessment \& } \\
\text { Rehabilitation }\end{array}$ & 2007 & Qualitative & $\begin{array}{l}\text { Leadership style, employer perception, } \\
\text { situational leadership }\end{array}$ \\
\hline Hashim, J., \& Saodah, W. & $\begin{array}{l}\text { Equality, Diversity and } \\
\text { Inclusion: An International } \\
\text { Journal }\end{array}$ & 2014 & Quantitative & $\begin{array}{l}\text { Employment, disability, organisational } \\
\text { behaviour }\end{array}$ \\
\hline $\begin{array}{l}\text { Hernandez, B., Chen, B., } \\
\text { Araten-Bergman, T., Levy, } \\
\text { J., Kramer, M., \& } \\
\text { Rimmerman, A. }\end{array}$ & $\begin{array}{l}\text { Employee Responsibilities } \\
\text { and Rights Journal }\end{array}$ & 2012 & $\begin{array}{l}\text { Empirical Study; } \\
\text { Qualitative Study }\end{array}$ & Non-profit, for profit, recruitment \\
\hline $\begin{array}{l}\text { Jasper, C. R., \& Waldhart, } \\
\text { P. }\end{array}$ & $\begin{array}{l}\text { International Journal of } \\
\text { Contemporary Hospitality } \\
\text { Management }\end{array}$ & 2013 & Qualitative & Recruitment, hospitality, attitudes \\
\hline Jones, P., \& Schmidt, R. A. & $\begin{array}{l}\text { International Journal of } \\
\text { Retail \& Distribution } \\
\text { Management }\end{array}$ & 2004 & Literature Review & Retail industry, disability \\
\hline $\begin{array}{l}\text { Kontosh, L. G., } \\
\text { Fletcher, I., Frain, M., } \\
\text { Winland-Brown, J. }\end{array}$ & $\begin{array}{l}\text { Work: A Journal of } \\
\text { Prevention, Assessment \& } \\
\text { Rehabilitation }\end{array}$ & 2007 & Quantitative & $\begin{array}{l}\text { Hiring practices, work place issues, } \\
\text { healthcare professionals }\end{array}$ \\
\hline Lee, B.A. & Industrial Relations & 2003 & Literature Review & $\begin{array}{l}\text { Americans with Disabilities Act (ADA), } \\
\text { policy, outcomes }\end{array}$ \\
\hline $\begin{array}{l}\text { Lengnick-Hall, M. L., } \\
\text { Gaunt, P. M., \& Kulkarni, } \\
\text { M. }\end{array}$ & $\begin{array}{l}\text { Human Resource } \\
\text { Management }\end{array}$ & 2008 & Qualitative & Disabilities; human resource; hiring \\
\hline $\begin{array}{l}\text { Nafukho, F. M., Roessler, R. } \\
\text { T., \& Kacirek, K. }\end{array}$ & $\begin{array}{l}\text { Advances in Developing } \\
\text { Human Resources }\end{array}$ & 2010 & $\begin{array}{l}\text { Preview of the special } \\
\text { issue }\end{array}$ & Diversity, disability, retention \\
\hline $\begin{array}{l}\text { Perry, E.L., Hendricks, W., } \\
\text { \& Broadbend, E. }\end{array}$ & Human Relations & 2000 & Qualitative & Discrimination, job satisfaction \\
\hline $\begin{array}{l}\text { Roessler, R., Hennessey, } \\
\text { M., Neath, J., Rumrill, P., \& } \\
\text { Nissen, S. }\end{array}$ & Journal of Rehabilitation & 2011 & Qualitative & $\begin{array}{l}\text { Multiple sclerosis, employment, } \\
\text { discrimination }\end{array}$ \\
\hline $\begin{array}{l}\text { Rumrill, P.D.J., \& } \\
\text { Fitzgerald, S.M. }\end{array}$ & $\begin{array}{l}\text { Advances in Developing } \\
\text { Human Resources }\end{array}$ & 2010 & Quantitative & Discrimination, disability laws, attitudes \\
\hline $\begin{array}{l}\text { Shier, M., Graham, J. R., \& } \\
\text { Jones, M. E. }\end{array}$ & Disability \& Society & 2009 & Qualitative & Employment, disability, discrimination \\
\hline $\begin{array}{l}\text { Schroedel, J. G., \& Geyer, } \\
\text { P. D. }\end{array}$ & $\begin{array}{l}\text { Journal of Applied } \\
\text { Rehabilitation Counselling }\end{array}$ & 2001 & Qualitative & $\begin{array}{l}\text { Career advancement, disability, hearing } \\
\text { loss }\end{array}$ \\
\hline $\begin{array}{l}\text { Schur, L., Kruse, D., \& } \\
\text { Blanck, P }\end{array}$ & $\begin{array}{l}\text { Behavioural Sciences \& the } \\
\text { Law }\end{array}$ & 2005 & Literature Review & Employment, culture, management \\
\hline \multicolumn{5}{|l|}{ Mental Health Issues } \\
\hline Cubero, G. & $\begin{array}{l}\text { Work: A Journal of } \\
\text { Prevention, Assessment \& } \\
\text { Rehabilitation }\end{array}$ & 2007 & Qualitative & $\begin{array}{l}\text { Workplace environment, leadership style, } \\
\text { situational leadership }\end{array}$ \\
\hline
\end{tabular}




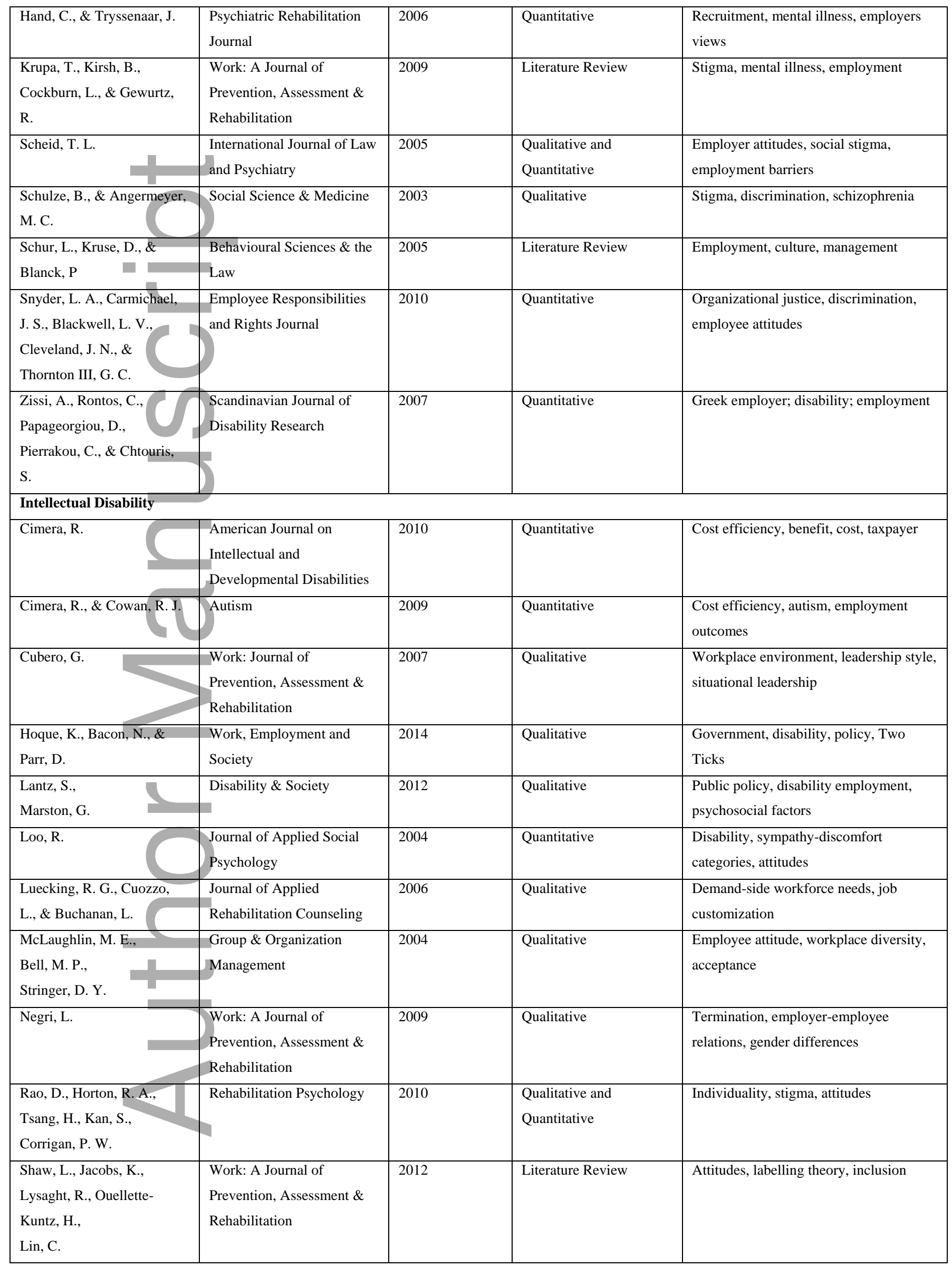




\section{Workers with disabilities and outcomes}

Research evidence demonstrates that workers with disabilities are generally successful employees. For example, workers with intellectual disabilities, given the appropriate training and support are often high performers (Unger \& Kregel, 2003). Supportive and inclusive management practices are positively associated with good work outcomes for people with disabilities (Gates, 2000; Lagerveld et al., 2010), and conversely inequitable and discriminatory treatment by managers/colleagues often leads to poor outcomes. Evidence suggests that workers with mental health problems tend to have more negative work experience than workers with physical disabilities.

\section{Workers with physical disabilities}

This section aims to examine the literature on the work related experiences and performance of workers with physical disabilities. There is some evidence to suggest positive management approaches increase an employee's chances of success in employment and subsequent career advancement (Schroedel \& Geyer, 2001). Schroedel and Geyer's study of 240 deaf and hearing impaired graduates reveals the majority of respondents were satisfied with their career progress. Supervisors were supportive, gave advice, praised their work and treated them fairly. However, individuals with physical disabilities may experience limited progression in their career as a result of their disability (Smith et al., 2004b; VillanuevaFlores, Valle-Cabrera, \& Bornay-Barrachina, 2014). Moreover, employees with a physical disability are more likely to be dissatisfied with their job when they perceive inequity and discrimination in their professional development opportunities.

Employers who have had experience with workers with physical disabilities generally describe them as cooperative and competent workers. Golub (2006) interviewed 22 employers from various sectors to explore their experiences and the key responsibilities of employers in respect of visually impaired workers. He found that when the organisation fully accepted workers with a physical disability there was a culture that embraced diversity as a strength and encouraged colleagues to focus on ability. Findings from Unger and Kregel's (2003) research demonstrate that supervisors within the retail, banking, healthcare, insurance, hospitality, technology and manufacturing sectors were generally pleased with the work performance of employees with physical disabilities. Supervisors gave higher ratings to 
punctuality, attendance and performance consistency but gave less desirable ratings on work speed. In this study it was noted that employee productivity was aligned to adequate and positive organisational support. Greenan, Wu and Black (2002) argue that employers should encourage colleagues to involve workers in all aspects of work life and social, non-work related events in order to foster an inclusive organisational culture.

\section{Workers with mental health issues}

The identified research articles indicate mental illness is more likely to impact on employee experiences and performance than other types of disability. In the first instance, potential employees, suffering with mental health problems, find their condition is a critical barrier to finding employment (Snyder et al., 2010). When they do find employment, the mental health of this cohort of workers often holds them back from achieving their performance and personal work goals.

A number of studies have examined the employee experiences and performance outcomes of workers with mental health problems. For example, research indicates that lost work time due to sick leave is significantly higher for employees suffering from depression compared to other employees. Stewart, Ricci, Chee, Hahn and Morganstein (2003) analysed 1,127 interviews from an American audit of productivity and depression. On average 5.6 hours per week were lost by employees suffering with depression, compared to 1.5 hours per week for employees with no reported condition. In the Lerner et al. (2004) study the overall rate of absenteeism due to depression was 1.7 days per week compared to 0.7 days for those without. The study suggests management needs to allow sufficient time for employees to perform their work, which will generate worker satisfaction, increase performance and reduce absenteeism.

Moreover, compared to other workers, workers with depression experience higher turnover rates (Lerner et al., 2004). MacDonald-Wilson, Rogers, Massaro, Lyass and Crean (2002) for example, conducted a multi-site qualitative study of 191 individuals with mental health issues in various entry-level jobs in the retail, services, wholesale, finance and manufacturing sectors across the USA. More than half of the respondents became unemployed within 12 months of appointment. Jones, Latreille and Slone (2006) examined Labour Force Survey data in the UK between 1997 and 2003, and also found a discrepancy in wages for workers with mental health issues (and other disabilities) relative to the general population. The sooner employers provide more support and equitable practices for workers with mental 
health issues the sooner workers will benefit from emotional support. Such assistance may well increase attendance, productivity and the retention of workers with mental health issues.

\section{Workers with intellectual disability}

Evidence suggests that employees with an intellectual disability are more likely to receive higher ratings of their work performance when employers perceive a good job-employee match (Gates, 2000; Lagerveld et al., 2010). For example, Smith, Webber, Graffam and Wilson (2004a) distributed surveys to 656 employers from various industries connected with disability employment services. The study found that employers rated workers with an intellectual disability as satisfactory when they met job expectations. Olson, Cioffi, Yovanoff and Mank (2001) suggest that employee confidence will be enhanced when employers consider them as capable of fulfilling their job duties. Martorell, Gutierrez-Recacha, Pereda and Ayuso-Mateos (2008) contend that personal and social factors such as self-determination can compensate for low intellectual functioning and facilitate employee performance.

To ensure positive work experiences, Lattimore, Parsons and Reid (2006) argue that pedagogical considerations such as extra tutorials must be afforded to workers with intellectual disabilities to increase skills and confidence. Their small case study of four workers with autism compared off-the-job training to on-the-job training. The researchers found that simulation training off-the-job produced a quicker skill uptake of workers. Offthe-job training gives workers the skills and confidence to perform and exposes them to the working environment with any potential organisational pressures. They suggest a combination of simulation off-the-job training and on-the-job training is needed to ensure workers with autism are job ready.

There is evidence in the US of the cost effectiveness of supported employment programmes for workers with intellectual disabilities. Cimera (2010) and Cimera and Cowan (2009) analysed US Vocational Rehabilitation Services supported employment program data from 104,213 workers with intellectual disabilities and 11,569 with autism, respectively. Cimera (2010) found that a worker with an intellectual disability generated a gross monthly benefit of \$769.54 for taxpayers which minus the supported employment operating costs gave a net benefit to the US tax payer \$133.10. Cimera and Cowan's (2009) study reveals that employment programme operating costs decreased by nine per cent from $\$ 3282$ per person in 2002 to $\$ 2992$ per person in 2006. 
Table 4 Performance and employment outcomes for workers with disabilities

\begin{tabular}{|c|c|c|c|c|}
\hline Author(s) & Journal & $\begin{array}{l}\text { Year of } \\
\text { Publication }\end{array}$ & $\begin{array}{l}\text { Data Collection } \\
\text { Method }\end{array}$ & Three Key Terms \\
\hline \multicolumn{5}{|l|}{ Physical Disability } \\
\hline Golub, D. B. & $\begin{array}{l}\text { Journal of Visual } \\
\text { Impairment \& Blindness }\end{array}$ & 2006 & Qualitative & Visual impairment, work experience \\
\hline $\begin{array}{l}\text { Greenan, J. P., Wu, M., \& } \\
\text { Black, E. L. }\end{array}$ & $\begin{array}{l}\text { Journal of Technology } \\
\text { Studies }\end{array}$ & 2002 & Quantitative & Disability, employment, employers \\
\hline $\begin{array}{l}\text { Schroedel, J. G., \& Geyer, } \\
\text { P. D. }\end{array}$ & $\begin{array}{l}\text { Journal of Applied } \\
\text { Rehabilitation Counselling }\end{array}$ & 2001 & Qualitative & $\begin{array}{l}\text { Career advancement, disability, hearing } \\
\text { loss }\end{array}$ \\
\hline $\begin{array}{l}\text { Smith, K., Webber, L., } \\
\text { Graffam, J., Wilson, C. }\end{array}$ & $\begin{array}{l}\text { Journal of Vocational } \\
\text { Rehabilitation }\end{array}$ & 2004 & Quantitative & $\begin{array}{l}\text { Employer satisfaction, strategic planning, } \\
\text { job performance }\end{array}$ \\
\hline $\begin{array}{l}\text { Villanueva-Flores, M., } \\
\text { Valle-Cabrera, R., \& } \\
\text { Bornay-Barrachina, M. }\end{array}$ & $\begin{array}{l}\text { Career Development } \\
\text { International }\end{array}$ & 2014 & Quantitative & $\begin{array}{l}\text { Perception, promotion, equal } \\
\text { opportunities }\end{array}$ \\
\hline Unger, D., \& Kregel J. & $\begin{array}{l}\text { Work: A Journal of } \\
\text { Prevention, Assessment \& } \\
\text { Rehabilitation }\end{array}$ & 2003 & $\begin{array}{l}\text { Qualitative and } \\
\text { quantitative }\end{array}$ & Accommodations, workplace support \\
\hline \multicolumn{5}{|c|}{ Mental Health Issues } \\
\hline $\begin{array}{l}\text { Jones, M. K., } \\
\text { Latreille, P. L., } \\
\text { Sloane, P. J. }\end{array}$ & Oxford Economic Papers & 2006 & Quantitative & Disability, gender differences, legislation \\
\hline $\begin{array}{l}\text { Lerner, D., Adler, D. A., } \\
\text { Chang, H., Lapitsky, L., } \\
\text { Hood, M. Y., Perissinotto, } \\
\text { C., Reed, J., McLaughlin, } \\
\text { T. J., Berndt, E. R., Rogers, } \\
\text { W. H }\end{array}$ & Psychiatric Services & 2004 & Quantitative & $\begin{array}{l}\text { Unemployment, job retention, } \\
\text { productivity }\end{array}$ \\
\hline $\begin{array}{l}\text { MacDonald-Wilson, K. L., } \\
\text { Rogers, E. S., Massaro, J. } \\
\text { M., Lyass, A., \& Crean, T. }\end{array}$ & $\begin{array}{l}\text { Community Mental health } \\
\text { Journal }\end{array}$ & 2002 & Quantitative & Accommodations, supported employment \\
\hline $\begin{array}{l}\text { Stewart, W. F., Ricci, J. A., } \\
\text { Chee, E., Hahn, S. R., \& } \\
\text { Morganstein, D. }\end{array}$ & $\begin{array}{l}\text { Journal of the American } \\
\text { Medical Association }\end{array}$ & 2003 & $\begin{array}{l}\text { Quantitative and } \\
\text { Qualitative }\end{array}$ & Productivity, depression, performance \\
\hline $\begin{array}{l}\text { Smith, K., Webber, L., } \\
\text { Graffam, J., \& Wilson, C. }\end{array}$ & $\begin{array}{l}\text { Journal of Vocational } \\
\text { Rehabilitation }\end{array}$ & 2004 & Quantitative & $\begin{array}{l}\text { Employer satisfaction, work } \\
\text { performance, hiring intentions }\end{array}$ \\
\hline $\begin{array}{l}\text { Snyder, L. A., Carmichael, } \\
\text { J. S., Blackwell, L. V., } \\
\text { Cleveland, J. N., \& } \\
\text { Thornton III, G. C. }\end{array}$ & $\begin{array}{l}\text { Employee Responsibilities } \\
\text { and Rights Journal }\end{array}$ & 2010 & Quantitative & $\begin{array}{l}\text { Organizational justice, discrimination, } \\
\text { employee attitudes }\end{array}$ \\
\hline \multicolumn{5}{|l|}{ Intellectual Disability } \\
\hline Cimera, R. & $\begin{array}{l}\text { American Journal on } \\
\text { Intellectual and } \\
\text { Developmental Disabilities }\end{array}$ & 2010 & Quantitative & Cost efficiency, benefit, cost, taxpayer \\
\hline Cimera, R., \& Cowan, R. J. & Autism & 2009 & Quantitative & $\begin{array}{l}\text { Cost efficiency, autism, employment } \\
\text { outcomes }\end{array}$ \\
\hline $\begin{array}{l}\text { Lagerveld, S. E., } \\
\text { Bültmann, U., Franche, R. }\end{array}$ & $\begin{array}{l}\text { Journal of Occupational } \\
\text { Rehabilitation }\end{array}$ & 2010 & Literature Review & $\begin{array}{l}\text { Occupational health and safety, } \\
\text { depression, sick leave }\end{array}$ \\
\hline
\end{tabular}




\begin{tabular}{|c|c|c|c|c|}
\hline $\begin{array}{l}\text { L., van Dijk, F. J., } \\
\text { Vlasveld, M. C., van der } \\
\text { Feltz-Cornelis, C. M., } \\
\text { Bruinvels, D. J., Huijs, J. } \\
\text { J., Blonk, R. W., van der } \\
\text { Klink, J. J., } \\
\text { Nieuwenhuijsen, K. }\end{array}$ & & & & \\
\hline $\begin{array}{l}\text { Lattimore, L. P., } \\
\text { Parsons, M. B., Reid, D. H. }\end{array}$ & $\begin{array}{l}\text { Journal of Applied } \\
\text { Behaviour Analysis }\end{array}$ & 2006 & Intervention study & $\begin{array}{l}\text { Vocational education, autism, supported } \\
\text { work }\end{array}$ \\
\hline $\begin{array}{l}\text { Martorell, A., Gutierrez- } \\
\text { Recacha, P., Pereda, A., \& } \\
\text { Ayuso-Mateos, J. L. }\end{array}$ & $\begin{array}{l}\text { Journal of Intellectual } \\
\text { Disability Research }\end{array}$ & 2008 & Quantitative & $\begin{array}{l}\text { Behavioural problems, intelligence } \\
\text { quotient, self-determination }\end{array}$ \\
\hline $\begin{array}{l}\text { Olson, D., Cioffi, A., } \\
\text { Yovanoff, P., \& Mank, D. }\end{array}$ & $\begin{array}{l}\text { Journal of Vocational } \\
\text { Rehabilitation }\end{array}$ & 2001 & Quantitative & $\begin{array}{l}\text { Employers perceptions, employment, } \\
\text { mental retardation }\end{array}$ \\
\hline $\begin{array}{l}\text { Smith, K., Webber, L., } \\
\text { Graffam, J., Wilson, C. }\end{array}$ & $\begin{array}{l}\text { Journal of Vocational } \\
\text { Rehabilitation }\end{array}$ & 2004 & Quantitative & $\begin{array}{l}\text { Employer satisfaction, work } \\
\text { performance, strategic planning }\end{array}$ \\
\hline
\end{tabular}

\section{Synthesis of themes}

In this section of the paper we provide an analysis of the core themes that have emerged from the scoping review.

\section{Knowledge and support}

\section{Physical disability}

The literature highlights low management knowledge and support around accommodations for workers with physical disabilities as a major issue. Employers are generally concerned about the appropriateness of any accommodations and how such accommodations will impact on their budget. Those employers who are willing to employ a person with a physical disability are generally prepared to provide apposite accommodations. Moreover, the complexity of such changes at the workplace raises integration issues. Colleagues could perceive that a person with a physical disability is receiving special treatment if, for example, they are provided with a modified or larger desk. Awareness training, around employees with physical disability, was found to be deficient for employers, managers and colleagues. Greater awareness can stimulate increased acceptance and those managers with knowledge and experience of working with people with physical disabilities are more likely to employ a person with a physical disability.

\section{Mental health disability}


Management knowledge of and experience in working with people with mental health disabilities was found to be positive towards the employment of people with this disability. The literature indicates that disclosure of a mental health disability is one of the greatest concerns for people with a disability. Workers fear that disclosing their disability may diminish their chances of securing employment and non-disclosure could negatively impact their disability if they do not seek out employer support. Employers and employees were found to be fearful of mental health disabilities due to the lack of disclosure and the unpredictable nature of some forms of mental health disability. Therefore, employers are concerned about creating a safe working environment for all employees.

\section{Intellectual disability}

Employers are less likely to employ a person with an intellectual disability due to inadequate knowledge and experience of intellectual disability. Employees with an intellectual disability are mostly employed in 'back of house' jobs and assigned menial and repetitive tasks. The accommodations for people with intellectual disabilities are different to workers with physical disabilities; they include shorter shift times, part-time hours and time off to attend medical appointments. Support is needed in different areas, such as customised job training, job design and additional mentoring. There is a lack of support for career prospects, with people with intellectual disabilities because they are generally assigned menial job roles. Nevertheless, there was a positive correlation with employers who have had experience interacting with people with intellectual disabilities and job placements.

\section{Commonalities and differences}

The knowledge and experience of employers of all types of disabilities was generally found to be deficient. However, for those who had the knowledge and experience there were positive outcomes. Modification to HR practices such as training and the alteration of job roles for people with disabilities are apparent in some organisations. The fear of the unknown concerning working with a person with a disability was found to be a precursor to the lack of employment for people with disabilities. The recruitment of people with different types of disabilities varied. People with physical disabilities are the most likely to find employment, followed by people with intellectual disabilities and those with mental health disabilities are the least likely to secure employment. Management attitudes and perceptions of the different types of disabilities and the training for staff and types of training needed for people with 
disabilities varied. Management tended to have greatest concern with the level of supervision and training required for those with an intellectual disability.

\section{Discrimination and Attitudes}

\section{Physical disability}

The literature indicates that management and employee stereotyping of workers with physical disabilities leads to perceptions of low productivity. Existing management experience working with a person with a physical disability positively influenced the attitudes of other management staff and employees. The more management and employees had worked with a person with a physical disability, the less stereotyping occurred. If managers had positive attitudes towards people with physical disabilities, then employees were more likely to have a positive attitude towards them.

\section{Mental Health disability}

People with mental health disabilities experience the most stigma at the workplace. Negative views and attitudes were predominant among management and employees due to scepticism of a disability, and the absence of clear symptoms (e.g., those with clinical depression). People with mental health disabilities are more prone to overt and covert discrimination.

\section{Intellectual disability}

The stigma surrounding intellectual disabilities influences the attitudes of managers and employees, which in turn impacts the workplace acceptance of people with intellectual disabilities. Again, any experience of working with a person with an intellectual disability was found to impact employer attitudes, with managers who have experience working with a person with an intellectual disability more likely to employ them. Social integration is a concern due to negative attitudes. The literature suggests that employers and employees are likely to distance themselves from people with intellectual disabilities and socially exclude them at work due to negative attitudes.

\section{Commonalities and differences}

The stigma surrounding disability impacts negatively on the acceptance of people with all types of disabilities at the workplace environment. The experience of employers and employees in interacting with people with disabilities engenders positive attitudes. However, the more experience a manager or employee had interacting with a person with a disability, 
the more accepting they were. It is common to find that workers with disabilities struggle to be socially included within the workplace. The levels of stigma across disabilities vary. The literature suggests that there is more stigma and less acceptance of workers with mental health disabilities compared to people with physical disabilities. Research suggests this is due to mental health disabilities not always being obvious and employers and employees disbelieving people with mental health disabilities when there are no apparent symptoms.

\section{Outcomes of employment of people with disabilities Physical disability}

Research indicates that low employment rates represent one of the main outcomes for people with physical disabilities. This is generally due to the lack of employer knowledge and support, as well as negative attitudes. There is an overall management expectation of poor outcomes for workers with physical disabilities. People with physical disabilities expect poor job outcomes and employers expect low levels of performance. Research suggests that best practice organisations perform well in integrating and supporting people with physical disabilities.

\section{Mental Health disability}

Mental health disabilities result in low employment rates due to the lack of employer knowledge and support and negative attitudes. Mental health disability appears to be the most challenging and expectations of poor outcomes for workers were the highest across the three disabilities. An outcome of this is high absenteeism and low retention rates. The literature illustrates that best practice organisations did well in integrating and supporting people with mental health disabilities.

\section{Intellectual disability}

The literature indicates there are management expectations of poor outcomes for workers with intellectual disabilities. This fosters low employment rates due to the lack of employer knowledge and support and negative attitudes towards people with intellectual disabilities. Best practice organisations did well in supporting people with intellectual disabilities.

\section{Commonalities and differences}

For all disability types the literature demonstrates low employment rates and the expectation of poor outcomes for all people with disabilities. This is predominantly due to the lack of 
employer knowledge and support and negative attitudes. Employers expect low performance outcomes and people with disabilities expect minor job roles with little scope for career progression. The literature illustrated that best practice organisations did well in integrating and supporting people with disabilities. The literature illustrated that people with mental health disabilities were the most challenging to support. This resulted in the lowest employment rates compared to intellectual and physical disabilities.

\section{Research Agenda}

Based on our scoping review of the literature relevant to HRM and management practices in respect of employment of people with disabilities we propose a research agenda to guide future research endeavours. It is evident that this is an under-researched, but also important topic with major economic, social and political implications for people with disabilities and the broader community. It is clear from the scoping review that HRM can play an important role in increasing the employment and success of workers with disabilities. To do this HRM reinforced by a variety of its functions (e.g., training and development of managers and employees) must shape, in partnership with its organisational citizens, the cultural values of the organisation to encourage and indeed celebrate diversity at the workplace. We suggest that management and disability researchers have an important role to play in encouraging the employment of people with disabilities.

First, there is an absence of systematic theory building in the area of HRM and people with disabilities. With the exception of a few notable studies, for example Hall et al., (2006) and Schur et al., (2009), the majority of research rests on small scale piece-meal studies aimed at describing current trends and issues regarding employment of people with disabilities. Suddaby, Hardy and Huy (2011) advocate the need for re-thinking theory building approaches in management research to capture the 'rich manifestation of organisations in society' and move beyond traditional conservative theory building practice. We support this and the need to unpack the organisational complexities that 'increasingly generates tensions, dualities, paradoxes, or contradiction in organisations' (Suddaby et al., 2011). HRM research does little to explain or provide solutions to the inherent paradox of the employment of people with disabilities - how do organisations promote workforce participation and opportunity whilst maximising employee performance and minimising accommodation costs? It is clear from the research above that people with disabilities in best practice organisations can and often do perform to a high level. We advocate theory-building that encompasses 
multiple bodies of literature and movement beyond knowledge silos, as well as combining epistemologies (Kilduff, Mehra, \& Dunn, 2011) and problematisation to challenge the implicit assumptions of existing theory and management approaches (Alvesson \& Sandberg, 2011).

Organisational studies offer a rich avenue for theory building on the links between HRM, disability and work. For example, Williams and Mavin (2012) argue that using intersectionality (the study of how social categories such as disability, race, class and gender intersect to shape peoples experiences) and connecting it with wider social, historical and political contexts will be important to understand how disability is constructed in organisations. They argue future studies need to traverse ableism and disability to break down intolerances and biased powers endemic in the workplace to enable the voices of workers with disability to be heard.

Second, there is an absence of representative large scale survey data on employer accommodation and management of workers with disabilities. To overcome knowledge silos, we suggest multi-disciplinary research teams (e.g., health, ergonomics, management and sociology) partnerships with peak management and HR bodies, government agencies and disability advocacy organisations to conduct large scale studies into management attitudes, current HR practices and barriers to the employment of people with disabilities. We also suggest the use of qualitative case studies on HR best practice to unpack the impact of effective and supportive HRM and associated outcomes for workers with disabilities. This approach is useful to breakdown myths around the employment of people with disabilities, to better understand and manage workplace challenges and promote the financial (e.g., business case for employment of people with disabilities) and social return on investment. Moreover, we need to know more about how organisations conceptualise and report on the effective outcomes of employing people with disabilities.

Third, the literature on HRM and management practices in relation to workers with disabilities is sparse and with the exception of relatively few articles, methodologically unsophisticated. Comprehensive study of the role of HRM in organisations is warranted. We recommend the study of HRM and workers with disabilities take into consideration different types of disabilities and levels of impairment and associated managerial responses. People 
with a disability are a diverse group and disability is a complex phenomenon, we suggest management study across a range of nations (e.g., developed and developing countries), industries, sectors and organisation forms (e.g., community groups) and cultural and ethnic groups. Moreover, further research into individual HRM functional areas such as human resource development, occupational health and safety, recruitment and selection, performance management, as well as leadership particularly line management is crucial to advance management understanding of how to support and create meaningful work for people with disabilities. Further examination of the antecedents of employer and managerial attitudes towards employing workers with disabilities is needed to advance the hiring of this cohort of workers.

Fourth, greater research is needed to better understand the support mechanisms provided by government and other agencies to organisations that employ people with disabilities (Australian Government, 2014) and how these services are used and related to outcomes for organisations and workers. It is also important to investigate the shortcoming of these services and how they can be improved for employers and employees. Further research is also required to unpack the various forms of work arrangements and organisational settings in which people with disabilities are employed. It is critical to identify settings that support the most effective outcomes for people with disabilities and understand what constitutes an 'effective outcome' from different perspectives. Employment support programs and the disclosure of mental health problems need to become a focal point for this research. More research is required into how government policy can support increased rates of employment of people with disabilities. Are greater government incentives or even quotas needed to grow the employment of people with disabilities?

Finally, methodological rigour and more complex research designs are required to enhance and improve the study of workers with disabilities. Further use of innovative theoretical approaches informed by cross-disciplinary research collaboration may offer new insights into old, as well as emerging challenges. Moreover, extending and developing deep partnerships as mentioned above will also facilitate developments in this research. For example, Metcalfe and Woodhams (2012: 136) argue that organisational studies need to broaden the scope of traditional diversity research (inclusive of disability) through expanding 'knowledge horizons across borders and territories and engage in interdisciplinary research endeavours'. They also add that scholars need to be attuned to 'intersecting processes at micro, meso and global levels of organisation and organising and how to develop management strategies to foster 
social justice, inclusiveness and equality' (Metcalfe and Woodhams, 2012: 136). Given the lack of quantitative studies on people with disability at work we would advocate that this is a rich avenue for research. In line with quantitative research approaches the use of multi-level and multi-response organisational/industry-based approaches are valuable to better understand the complexities of organisational, political and contextual realities of employing people with disabilities. HR researchers must consider using more complex research approaches such as experimental research designs with training and organizational change interventions and studies that use panel data and longitudinal designs to assess the outcomes of employing people with disabilities for employers, workers and the wider community.

\section{Conclusion}

This scoping review has provided an evidence-based approach to contemporary literature on HR and management practices in the disability sector. The review set out to provide an overview of organisational practices and accumulate evidence about HR and management practices and attitudes that impact on workers with physical, mental health and intellectual disabilities. By taking this approach we were able to outline our systematic methods, identify gaps in the research, inform management scholars about this critical area of management, and develop a research agenda.

Based on our literature review, we make four concluding points. First, there is a lack of systematic research and academic rigor in the HRM and management research on people with disabilities. Much of the research is generalised and does not distinguish between people with different types or levels of impairment. The research tends to over-simplify the complexity of disability. Second, little attention has been paid to theory-building and new ways of conceptualising the role of HRM in the disability sector. Third, there is substantial evidence of widespread discrimination among managers and poor use of HRM by organisations. There is a disconnection between HRM policies and practices and support for employees with disabilities (Kulkarni \& Valk, 2010). Discrimination in the workplace impacts on employees with a disability through negative employer perceptions, less opportunities for promotion (Schur et al., 2005), stricter standards of performance (Graffam et al., 2002), forced supervision and failure to appoint due to the disability (Shier et al., 2009). More precisely, the research indicates that workers with intellectual disabilities have more negative experiences than those with physical or mental health issues (Negri, 2009; Rao et al., 2010), but more positive experiences if a manager has had experience in managing a worker with an 
intellectual disability (Cunningham et al., 2004). The management support that does exist appears to be limited to structural accommodations rather than the personal or professional support employees with disabilities need (McDowell \& Fossey, 2015). Fourth, it is clear from the literature that effective HRM and supervisory support for workers with disabilities is positively associated with employee wellbeing, career success and employee performance (Yang \& Konrad, 2011).

In conclusion, it is evident from this review that there is a need for further research on the role of HRM and management practices in relation to disability in order that employers are more inclusive of employees with disabilities. Researchers and scholars can support managers through more theoretically and methodologically robust research that informs government policy, and HRM and management practice. The key to improving the support of people with disabilities at work, increasing their employment and social inclusion, is partnership between government, schools, business, disability advocate groups, academe and workers themselves.

\section{Reference List}

Almond, S., \& Healey, A. 2003. Mental health and absence form work: New evidence from the UK quarterly labour force survey Work Employment and Society, 17(4): 731-742.

Alston, R., Bell, T., \& Hampton, J. 2002. Learning disability and career entry into the sciences: A critical analysis of attitudinal factors. Journal of Career Development (Springer Science \& Business Media B.V.), 28(4): 263-275.

Alvesson, M., \& Sandberg, J. 2011. Generating research questions through problematization. Academy of Management Review, 36(2): 247-271.

Australian Bureau of Statistics. 2012. Disability, Ageing and Carers.

Australian Government. 2014. Job Access: An Australian Government initiative, Vol. 2014.

Ball, P., Monaco, G., Schmeling, J., Schartz, H., \& Blanck, P. 2005. Disability as diversity in Fortune 100 companies. Behavioral Sciences \& the Law, 23(1): 97-121.

Balser, D. B. 2002. Agency in organizational inequality: Organizational behavior and individual perceptions of discrimination. Work and Occupations, 29(2): 137-165.

Barlow, J. H., Wright, C. C., \& Wright, S. 2003. Development of job-seeking ability in people with arthritis: evaluation of a pilot program. International Journal of Rehabilitation Research, 26(4): 329-333.

Bassett, J., Lloyd, C., \& Bassett, H. 2001. Work issues for young people with psychosis: Barriers to employment. British Journal of Occupational Therapy, 64(2): 66-72.

This article is protected by copyright. All rights reserved 
Bengisu, M., \& Balta, S. 2011. Employment of the workforce with disabilities in the hospitality industry. Journal of Sustainable Tourism, 19(1): 35-57.

Bigby, C., \& Frawley, P. 2010. Reflections on doing inclusive research in the 'Making Life Good in the Community' study. Journal of Intellectual and Developmental Disability 35(2): 53-61.

Chan, F., Strauser, D., Maher, P., Lee, E., Jones, R., \& Johnson, E. T. 2010. Demand-side factors related to employment of people with disabilities: A survey of employers in the midwest region of the United States. Journal of Occupational Rehabilitation, 20(4): 412-419.

Charmaz, K. 2010. Disclosing illness and disability in the workplace. Journal of International Education in Business, 3(1/2): 6-19.

Cimera, R. 2010. National cost efficiency of supported employees with intellectual disabilities: 2002 to 2007 American Journal on Intellectual and Developmental Disabilities, 115(1): 19-29.

Cimera, R., \& Cowan, R. J. 2009. The costs of services and employment outcomes achieved by adults with autism in the US. Autism, 13(3): 285-302.

Cook, J. A., \& Burke-Miller, J. K. 2015. Reasons for job separations in a cohort of workers with psychiatric disabilities. Journal of Rehabilitation Research \& Development, 52(4): 371-384.

Cubero, C. G. 2007. Situational leadership and persons with disabilities. Work: Journal of Prevention, Assessment \& Rehabilitation, 29(4): 351-356.

Cunningham, I., James, P., \& Dibben, P. 2004. Bridging the gap between rhetoric and reality: Line managers and the protection of job security for ill workers in the modern workplace. British Journal of Management, 15(3): 273-290.

Donelly, M., \& Given, F. 2010. Employment programs and professionals with a disability. Work, 36(2): 217-225.

Duff, A., \& Ferguson, J. 2007. Disability and accounting firms: Evidence from the UK. Critical Perspectives on Accounting, 18(1): 139-157.

Fevre, R., Robinson, A., Lewis, D., \& Jones, T. 2013. The ill treatment of employees with disabilities in British workplaces. Work Employment and Society, 27(2): 288-307.

Fillary, R., \& Pernice, R. 2006. Social inclusion in workplaces where people with intellectual disabilities are employed: implications for supported employment professionals. International Journal of Rehabilitation Research, 29(1): 31-36.

Fyffe, C. (Ed.). 2007. Understanding intellectual disabilities. London: Jessica Kingsley.

Gates, L. B. 2000. Workplace accommodation as a social process. Journal of Occupational Rehabilitation, 10(1): 85-98.

Golub, D. B. 2006. A model of successful work experience for employees who are visually impaired: the results of a study. Journal of Visual Impairment \& Blindness, 100(12): 715-725.

This article is protected by copyright. All rights reserved 
Graffam, J., Shinkfield, A., Smith, K., \& Polzin, U. 2002. Factors that influence employer decisions in hiring and retaining an employee with a disability. Journal of Vocational Rehabilitation, 17(3): 175-181.

Greenan, J. P., Wu, M., \& Black, E. L. 2002. Perspectives on employing individuals with special needs. Journal of Technology Studies, 28(1/2): 29-37.

Groschl, S. 2007. An exploration of HR policies and practices affecting the integration of persons with disabilities in the hotel industry in major Canadian tourism destinations. International Journal of Hospitality Management, 26(3): 666-686.

Hall, A. C., Timmons, J. C., Boeltzig, H., Hamner, D., \& Fesko, S. 2006. Maximizing competence through professional development: Increasing disability knowledge among One-Stop Career Center staff. Work, 26(4): 355-367.

Hamner, D., Hall, A. C., Timmons, J. C., Boeltzig, H., \& Fesko, S. 2008. Agents of change in the disability field: Bridge-builders who make a difference. Journal of Organizational Change Management, 21(2): 161-173.

Hand, C., \& Tryssenaar, J. 2006. Small business employers' views on hiring individuals with mental illness. Psychiatric Rehabilitation Journal, 29(3): 166-173.

Hartnett, H. P., Stuart, H., Thurman, H., Loy, B., \& Batiste, L. C. 2011. Employers' perceptions of the benefits of workplace accommodations: Reasons to hire, retain and promote people with disabilities. Journal of Vocational Rehabilitation, 34(1): 17-23.

Hashim, J., \& Saodah, W. 2014. Predictors to employees with disabilities' organisational behaviour and involvement in employment. Equality, Diversity and Inclusion: An International Journal, 33(2): 193-209.

Hernandez, B., Chen, B., Araten-Bergman, T., Levy, J., Kramer, M., \& Rimmerman, A. 2012. Workers with disabilities: Exploring the hiring intentions of nonprofit and for-profit employers. Employee Responsibilities and Rights Journal, 24(4): 237-249.

Hoque, K., Bacon, N., \& Parr, D. 2014. Employer disability practice in Britain: Assessing the impact of the positive about disabled people 'Two Ticks' symbol. Work, Employment and Society, 28(3): 430-451.

Houtenville, A., \& Kalargyrou, V. 2012. People with disabilities: Employers' perspectives on recruitment practices, strategies, and challenges in leisure and hospitality. Cornell Hospitality Quarterly, 53(1): 40-52.

Jasper, C. R., \& Waldhart, P. 2013. Employer attitudes on hiring employees with disabilities in the leisure and hospitality industry. International Journal of Contemporary Hospitality Management, 25(4): 577-594.

This article is protected by copyright. All rights reserved 
Jones, M., Latreille, P., \& Sloane, L. 2006. Disability, gender, and the British labour market. Oxford Economic Papers, 58(3): 407-449.

Jones, P., \& Schmidt, R. A. 2004. Retail employment and disability. International Journal of Retail \& Distribution Management, 32(8): 426-429.

Kaye, H. S., Jans, L. H., \& Jones, E. C. 2011. Why don't employers Hire and retain workers with disabilities? Journal of Occupational Rehabilitation, 21(4): 526-536.

Kilduff, M., Mehra, A., \& Dunn, M. B. 2011. From blue sky research to problem solving: A philosophy of science theory of new knowledge production. Academy of Management Review, 36(2): 297-317.

Kontosh, L. G., Fletcher, I., Frain, M., \& Winland-Brown, J. 2007. Work place issues surrounding healthcare professionals with disabilities in the current labor market. Work: Journal of Prevention, Assessment \& Rehabilitation, 29(4): 295-302.

Krupa, T., Kirsh, B., Cockburn, L., \& Gewurtz. 2009. Understanding the stigma of mental illness in employment. Work, 33: 413-425.

Kulkarni, M., \& Valk, R. 2010. Don't ask, don't tell: Two views on human resource practices for people with disabilities. IIMG Management Review, 22(1): 137-146.

Lagerveld, S. E., Bültmann, U., Franche, R. L., van Dijk, F. J., Vlasveld, M. C., van der Feltz-Cornelis, C. M., Bruinvels, D. J., Huijs, J. J., Blonk, R. W., van der Klink, J. J., \& Nieuwenhuijsen, K. 2010. Factors associated with work participation and work functioning in depressed workers: A systematic review. Journal of Occupational Rehabilitation, 20(3): 275-292.

Lantz, S., \& Marston, G. 2012. Policy, citizenship and governance: The case of disability and employment policy in Australia. Disability \& Society, 27(6): 853-867.

Lattimore, L. P., Parsons, M. B., \& Reid, D. H. 2006. Enchanting job-site training of supported workers with autism: A reemphasis on simulation. Journal of Applied Behavior Analysis, 39(1): 91102

Lee, B. A. 2003. A decade of the Americans with Disabilities Act: Judicial outcomes and unresolved problems. Industrial Relations, 42(1): 11-30.

Lengnick-Hall, M., Gaunt, P. M., \& Kulkarni, M. 2008. Overlooked and underutilised: People with disabilities are an untapped human resource. Human Resource Management Review, 47(2): 255-273.

Lerner, D., Adler, D. A., Chang, H., Lapitsky, L., Hood, M. Y., Perissinotto, C., Reed, J., McLaughlin, T. J., Berndt, E. R., \& Rogers, W. H. 2004. Unemployment, job retention, and productivity loss among employees with depression. Psychiatric Services, 55(12): 1371-1378.

This article is protected by copyright. All rights reserved 
Lewis, G., Thoresen, S. H., \& Cocks, E. 2011. Successful approaches to placing and supporting apprentices and trainees with disability in Australia. Journal of Vocational Rehabilitation, 34(3): 181-189.

Lewis, R., Dobbs, L., \& Biddle, P. 2013. 'If this wasn't here I probably wouldn't be': Disabled workers' views of employment support. Disability \& Society, 28(8): 1089-1103.

Lexen, A., Hofgren, C., \& Bejerholm, U. 2013. Reclaiming the worker role: Perceptions of people with mental illness participating in IPS. Scandinavian Journal of Occupational Therapy, 20: 54-63.

Loo, R. 2004. Attitudes toward employing persons with disabilities: A test of the sympathydiscomfort categories. Journal of Applied Social Psychology, 34(10): 2200-2214.

Luecking, R. G., Cuozzo, L., \& Buchanan, L. 2006. Demand-side workforce needs and the potential for job customization. Journal of Applied Rehabilitation Counseling, 37(4): 5-13.

Lysaght, R., Quellette-Kuntz, H., \& Lin, C.-J. 2012. Untapped potential: Perspectives on the employment of people with intellectual disability. Work: A Journal of Prevention, Assessment and Rehabilitation, 41: 409-422.

MacDonald-Wilson, K. L., Rogers, E. S., Massaro, J. M., Lyass, A., \& Crean, T. 2002. An investigation of reasonable workplace accommodations for people with psychiatric disabilities: Quantitative findings from a multi-site study. Community Mental Health Journal, 38: 35-50.

Martorell, A., Gutierrez-Recacha, P., Pereda, A., \& Ayuso-Mateos, J. L. 2008. Identification of personal factors that determine work outcomes for adults with intellectual disability. Journal of Intellectual Disability Research, 52(12): 1091-1101.

McDowell, C., \& Fossey, E. 2015. Workplace Accommodations for People with Mental Illness: A Scoping Review. Journal of occupational rehabilitation, 25(1): 197-206.

McLaughlin, M. E., Bell, M. P., \& Stringer, D. Y. 2004. Stigma and acceptance of persons with disabilities: Understudied aspects of workforce diversity. Group \& Organization Management, 29(3): 302-333.

Mumby, D. K. 2008. Theorising the future of critical organization studies. In D. Barry, \& H. Hansen (Eds.), The Sage Handbook of New Approaches in Management and Organization: 27-28. Los Angeles, CA: Sage.

Nafukho, F. M., Roessler, R. T., \& Kacirek, K. 2010. Disability as a diversity factor: Implications for human resource practices. Advances in Developing Human Resources, 12(4): 395.

Negri, L. 2009. Why has a woman with mental illness been fired? Discourses on fairness and diversity management. Work, 33(1): 35-42.

OECD. 2010. Sickness, Disability and Work: Breaking the Barriers: A synthesis of findings across OECD countries. : OECD Publishing. 
Olson, D., Cioffi, A., Yovanoff, P., \& Mank, D. 2001. Employers' perceptions of employees with mental retardation. Journal of Vocational Rehabilitation, 16: 125-133.

Perry, E. L., Hendricks, W., \& Broadbend, E. 2000. An exploration of access and treatment discrimination and job satisfaction among college graduate with and without physical disabilities. Human Relations, 53(7): 923-955.

Price, L. A., \& Gerber, P. J. 2001. At second glance: Employers and employees with learning disabilities in the Americans with Disabilities Act era. Journal of Learning Disabilities, 34(3): 202-210, 248.

Rao, D., Horton, R. A., Tsang, H., Kan, S., \& Corrigan, P. W. 2010. Does individualism help explain differences in employers' stigmatizing attitudes toward disability across Chinese and American cities? Rehabilitation Psychology, 55(4): 351-359.

Roessler, R., Hennessey, M., Neath, J., Rumrill, P., \& Nissen, S. 2011. The employment discrimination experiences of adults with multiple sclerosis. Journal of Rehabilitation, 77(1): 20-30.

Roessler, R. T., Hurley, J. E., \& McMahon, B. T. 2010. A comparison of allegations and resolutions involving issues of discharge versus constructive discharge: Implications for diversity management. Advances in Developing Human Resources, 12(4): 407-428.

Rosenheck, R., Leslie, D., Keefe, R., McEvoy, J., Swartz, M., Perkins, D., Stroup, S., Hsiao, J. K., \& Lieberman, J. 2006. Barriers to employment for people with schizophrenia. American Journal of Psychiatry, 163(3): 411-417.

Roush, S. 2009. The Menu Approach to supported employment for individuals with severe and persistent mental illness: Outcomes in an Oregon community based program. Work, 34(1): 45-51.

Rumrill, P. D., Jr., \& Fitzgerald, S. M. 2010. Employer characteristics and discharge-related discrimination against people with disabilities under the Americans With Disabilities Act. Advances in Developing Human Resources, 12(4): 448-465.

Sakai, K., Hashimoto, T., \& Inuo, S. 2009. Factors associated with work outcome among individuals with schizophrenia: Investigating work support in Japan. Work, 32(2): 227.

Salkever, D. S., Karakus, M. C., Slade, E. P., Harding, C. M., Hough, R. L., Rosenheck, R. A., Swartz, M. S., Barrio, C., \& Yamada, A. M. 2007. Measures and predictors of community-based employment and earnings of persons with schizophrenia in a multisite study. Psychiatric Services, 58(3): 315-324.

Scheid, T. L. 2005. Stigma as a barrier to employment: Mental disability and the Americans with Disabilities Act. International Journal of Law and Psychiatry, 28(6): 670-690. 
Schmidt, M. A., \& Smith, D. L. 2007. Individuals with disabilities perceptions on preparedness for the workforce and factors that limit employment. Work, 28(1): 13-21.

Schroedel, J. G., \& Geyer, P. D. 2001. Enhancing the career advancement of workers with hearing loss: Results from a national follow-up survey. Journal of Applied Rehabilitation Counseling, 32(3): 35-44.

Schulze, B., \& Angermeyer, C. 2003. Subjective experiences of stigma. A focus group study of schizophrenic patients, their relatives and mental health professionals. Social Science \& Medicine, 56: 299-312.

Schur, L., Kruse, D., \& Blanck, P. 2005. Corporate culture and the employment of persons with disabilities. Behavioral Sciences \& the Law, 23(1): 3-20.

Schur, L., Kruse, D., Blasi, J., \& Blanck, P. 2009. Is disability disabling in all workplaces? Workplace disparities and corporate culture. Industrial Relations, 48(3): 381-410.

Shaw, L., Jacobs, K., Lysaght, R., Ouellette-Kuntz, H., \& Lin, C.-J. 2012. Untapped potential: Perspectives on the employment of people with intellectual disability. Work, 41(4): 409-422.

Shier, M., Graham, J. R., \& Jones, M. E. 2009. Barriers to employment as experienced by disabled people: A qualitative analysis in Calgary and Regina, Canada. Disability \& Society, 24(1): 6375.

Smith, K., Webber, L., Graffam, J., \& Wilson, C. 2004a. Employer satisfaction with employees with a disability: Comparisons with other employees. Journal of Vocational Rehabilitation, 21(2): 61-69.

Smith, K., Webber, L., Graffam, J., \& Wilson, C. 2004b. Employer satisfaction, job-match and future hiring intentions for employees with a disability. Journal of Vocational Rehabilitation, 21(3): 165-173.

Snyder, L., Carmichael, J., Blackwell, L., Cleveland, J., \& Thornton, G. 2010. Perceptions of discrimination and justice among employees with disabilities. Employee Responsibilities \& Rights Journal, 22(1): 5-19.

Stewart, W. F., Ricci, J. A., Chee, E., Hahn, S. R., \& Morganstein, D. 2003. Cost of lost productive work time among US workers with depression. JAMA, 289(3): 3135-2128.

Suddaby, R., Hardy, C., \& Huy, Q. N. 2011. Where are the new theories of organization? Academy of Management Review, 36(2): 236-246.

Tsang, H. W. H., Angell, B., Corrigan, P. W., Lee, Y., Shi, K., Lam, C. S., Jin, S., \& Fung, K. M. T. 2007. A cross-cultural study of employers' concerns about hiring people with psychotic disorder: Implications for recovery. Social Psychiatry and Psychiatric Epidemiology 42(9): 723-733.

This article is protected by copyright. All rights reserved 
Unger, D., \& Kregel, J. 2003. Employers' knowledge and utilization of accommodations. Work: Journal of Prevention, Assessment \& Rehabilitation, 21(1): 5-15.

United Kingdom Government. 2012. Labour Force Survey. In O. f. N. Statistics (Ed.).

Villanueva-Flores, M., Valle-Cabrera, R., \& Bornay-Barrachina, M. 2014. Career development and individuals with physical disabilities. Career Development International, , 19(2): 222-243. World Health Organization. 2001. International Classification of functioning, Disability and Health (ICF). Geneva, Switzerland: World Health Organization.

World Health Organization. 2002-2004. World Health Survey. Geneva: World Health Organization. Yang, Y., \& Konrad, A. I. M. 2011. Understanding diversity management practices: Implications of institutional theory and resource-based theory Group Organization Management, 36(1): 638.

Zissi, A., Rontos, C., Papageorgiou, D., Pierrakou, C., \& Chtouris, S. 2007. Greek employers' attitudes to employing people with disabilities: Effects of the type of disability. Scandinavian Journal of Disability Research, 9: 14-25.

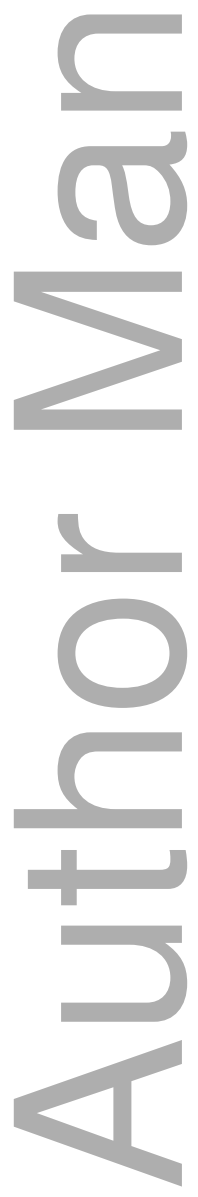

This article is protected by copyright. All rights reserved 


\section{University Library}

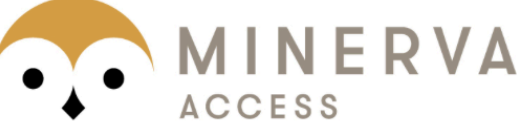

A gateway to Melbourne's research publications

Minerva Access is the Institutional Repository of The University of Melbourne

Author/s:

Cavanagh, J;Bartram, T;Meacham, H;Bigby, C;Oakman, J;Fossey, E

Title:

Supporting workers with disabilities: a scoping review of the role of human resource management in contemporary organisations

Date:

2017-01-01

Citation:

Cavanagh, J., Bartram, T., Meacham, H., Bigby, C., Oakman, J. \& Fossey, E. (2017). Supporting workers with disabilities: a scoping review of the role of human resource management in contemporary organisations. ASIA PACIFIC JOURNAL OF HUMAN RESOURCES, 55 (1), pp.6-43. https://doi.org/10.1111/1744-7941.12111.

Persistent Link:

http://hdl.handle.net/11343/291317 